TIT/HEP-535

RIKEN-TH-38

UT-05-03

hep-th/0503033

March, 2005

\title{
Global Structure of Moduli Space for BPS Walls
}

\author{
Minoru Eto ${ }^{1}$, Youichi Isozumi ${ }^{1}$, Muneto Nitta $^{1}$, Keisuke Ohashi $^{1}$, \\ Kazutoshi Ohta ${ }^{2}$, Norisuke Sakai ${ }^{1}$ and Yuji Tachikawa ${ }^{3}$ \\ ${ }^{1}$ Department of Physics, Tokyo Institute of Technology \\ Tokyo 152-8551, JAPAN \\ 2 Theoretical Physics Laboratory \\ The Institute of Physical and Chemical Research (RIKEN) \\ Saitama 351-0198, JAPAN \\ ${ }^{3}$ Department of Physics, University of Tokyo \\ Tokyo 112-0033, JAPAN
}

\begin{abstract}
We study the global structure of the moduli space of BPS walls in the Higgs branch of supersymmetric theories with eight supercharges. We examine the structure in the neighborhood of a special Lagrangian submanifold $M$, and find that the dimension of the moduli space can be larger than that naively suggested by the index theorem, contrary to previous examples of BPS solitons. We investigate BPS wall solutions in an explicit example of $M$ using Abelian gauge theory. Its Higgs branch turns out to contain several special Lagrangian submanifolds including $M$. We show that the total moduli space of BPS walls is the union of these submanifolds. We also find interesting dynamics between BPS walls as a byproduct of the analysis. Namely, mutual repulsion and attraction between BPS walls sometimes forbid a movement of a wall and lock it in a certain position; we also find that a pair of walls can transmute to another pair of walls with different tension after they pass through.
\end{abstract}

e-mail addresses: meto,isozumi,nitta,keisuke@th.phys.titech.ac.jp; k-ohta@riken.jp; nsakai@th.phys.titech.ac.jp; yujitach@hep-th.phys.s.u-tokyo.ac.jp 


\section{Introduction}

Solitons play a key role in understanding non-perturbative dynamics in quantum field theories. In supersymmetric (SUSY) theories, the Bogomol'nyi-Prasad-Sommerfield (BPS) saturated solitons preserve a part of SUSY [1, 2, and they are important in understanding the quantum correction to perturbatively non-renormalized quantities. BPS solitons in SUSY theories are also important in the brane-world scenario [3]- $[5]$, because they are the necessary ingredients to build SUSY models on their worldvolume with non-singular space-time.

BPS solitons usually contain a number of parameters. These parameters are called moduli, which provide massless degrees of freedom in low-energy effective Lagrangians on the worldvolume of the solitons. The kinetic term for them are nontrivial, and that makes the moduli spaces for the BPS solitons fascinating subjects. The BPS solitons in SUSY (possibly gauge) theories with codimension four, three, two, and one are called instantons, monopoles, vortices, and domain walls, respectively. The construction of the moduli space for instantons are well-established by Atiyah, Drinfeld, Hitchin, and Manin 6 . and for monopoles by Nahm [7]. These results have found many physical and mathematical applications. The moduli space of vortices has also been constructed recently by using the D-brane configurations [8, 9], after the discovery for the case of one vortex [10].

Somewhat surprisingly, moduli spaces of domain walls were relatively unexplored for a long time although they are the simplest of such solitons. We have seen that much attention has been paid to BPS domain walls in these years [11-14 as a revival of studies in more than ten years ago [15, 16]. One of their motivation is the relation to the brane-world scenario. We should construct BPS walls in SUSY theories with eight supercharges for application to the SUSY brane-world. BPS walls and other BPS solitons in SUSY gauge theories or SUSY nonlinear sigma models (NLSM) with eight supercharges have been studied extensively [16]-36]. Models and soliton solutions in the latter can be obtained by those in the former by taking the limit of strong gauge coupling, and it is often far easier to obtain (exact) solutions in the latter than the former. The SUSY NLSM with eight supercharges should have the hyper-Kähler target manifold [37]-[42]. The method to obtain hyper-Kähler manifolds by taking the strong gauge coupling limit in gauge theories is called the hyper-Kähler quotient construction [39]-[41, and it can sometimes yield a target manifold of the form of $T^{*} M$ with a Kähler manifold $M$ as its special Lagrangian submanifold.

The moduli space of BPS walls was considered in a $d=4, \mathcal{N}=1$ generalized WessZumino model [12] and in $d=4, \mathcal{N}=1$ SUSY QCD [13, 14]. However, for SUSY theories with eight supercharges, the moduli space of BPS walls was constructed only for $U(1)$ gauge theory [19, 20] until recently. The moduli space of BPS walls has been determined completely in the case of $U\left(N_{c}\right)$ gauge theories with massive $N_{f}\left(>N_{c}\right)$ fundamental flavors with eight supercharges [28, 29]. It has been found that the moduli spaces for various topological sectors glue together to form a complex Grassmannian $M=G r_{N_{f}, N_{c}} \simeq$ $S U\left(N_{f}\right) /\left[S U\left(N_{c}\right) \times S U\left(N_{f}-N_{c}\right) \times U(1)\right]$, which is a special Lagrangian submanifold of the Higgs branch $T^{*} G r_{N_{f}, N_{c}}$ of the corresponding massless model. This total moduli space 
is also valid for the massive hyper-Kähler NLSM on $T^{*} G r_{N_{f}, N_{c}}$, which can be obtained by taking the strong coupling limit of the gauge theories. Hence it is very natural to ask whether the total moduli space of BPS walls in $T^{*} M$ is always $M$.

The domain walls enjoy also several interesting properties [29]. For instance, there are mutually penetrable pairs of walls which can pass through each other by changing the sign of the modulus for their relative distance. Up to now it has been found that each of these walls maintain its individual identity after passing through. On the other hand, certain pairs of walls are mutually impenetrable and produces a single compressed wall, when the modulus for their relative distance is taken to minus infinity. So far in previous works, we find that all pairs of walls are impenetrable in Abelian gauge theories and in the NLSM resulting from it.

The purpose of this paper is to investigate the global structure of the moduli space of BPS walls in the Higgs branch of SUSY theories with eight supercharges. By considering the local structure around a special Lagrangian submanifold $M$, we first find that the dimension of the moduli space can be larger than that naively indicated by the index theorem. To our knowledge, this sort of phenomenon of additional zero modes has not been found in the case of other BPS solitons. Taking an Abelian gauge theory to obtain a NLSM for $T^{*} M$ as an explicit example, we work out BPS wall solutions. Several special Lagrangian submanifolds $M_{a}$ 's besides $M$ are found to be contained in its Higgs branch. We find that the total moduli space of the BPS walls is the union of these special Lagrangian submanifolds $M_{a}$ 's, some of which share boundary subspaces. Thus the total moduli space gains very ample structure contrary to naive expectation. The hyper-Kähler quotient itself is given by gluing $T^{*} M_{a}$ 's together.

By considering the physical reason behind the BPS wall moduli space, we also observe an interesting mutual repulsion and attraction between BPS domain walls. We also find a new phenomenon where a pair of walls can pass through each other, resulting in a different pair of walls. Namely a pair of walls are transmuted to another pair of walls with different tension while preserving the total tension after their encounter. We recognize that penetrable pairs of walls are not restricted to non-Abelian gauge theories (and its associated NLSM). The penetrable pairs can also occur in gauge theories with rank larger than unity, such as a direct product of $U(1)$ factors. In particular, it is quite common to have pairs of walls which transmute through crossing when there are two or more factors of $U(1)$ gauge groups. We will see that the occurrence of these phenomena is intimately related to the existence of multiple compact Lagrangian submanifolds, which is also responsible for the violation of transversality.

Before we move on to the main part, we would like to comment on the nature of our analysis. We only analyze classical aspects of BPS domain walls in the main part of our paper. Due to the high degree of SUSY, the quantum correction is under better control than those with less supercharges. Furthermore, the target spaces we treat in the latter half of our paper have as many tri-holomorphic $U(1)$ isometries as possible. Hence, they should give us a firm grip on the correction to the hyper-Kähler metric, although that for the general Higgs branch is relatively unexplored compared to that for the Coulomb branch. Effective theories on the BPS walls, on the other hand, have only four supercharges, which 
implies that there will be much more diverse interesting non-perturbative phenomena. We plan to discuss these matters in a future publication.

The organization of the paper is as follows: We discuss in section 2 the BPS flow equation in $T^{*} M$, and study the relation between the dimension of the moduli space and the index theorem. We then move on to study in section 3 the BPS domain walls in hypertoric manifolds, 1.e. the Higgs branch of gauged linear sigma models. There the reader will find explicit and concrete examples displaying phenomena uncovered in section 2, We will see how several toric Kähler manifolds glue together to form the total moduli space of BPS walls. We also find interesting dynamics among BPS walls, using the techniques developed in the previous sections. We conclude the paper in section 4 with some discussion. There are two appendices. Appendix A will serve as a collection of definitions concerning the Morse-Smale transversality. In appendix B, we analyze the index and vanishing theorem from the viewpoint of gauged linear sigma models.

\section{BPS flows and the index theorem}

\subsection{BPS flows on general hyper-Kähler manifolds}

First we give a brief review of the BPS domain wall on general massive hyper-Kähler sigma models. We consider theories with eight supercharges, which force us to take a hyper-Kähler manifold as the target space of the NLSM [37. Hyper-Kähler manifolds of real dimension $4 n$ are Riemannian manifolds with the holonomy group reduced to $S p(n)$. In other words, there are three complex structures $I_{a}(a=1,2,3)$, satisfying the relation of imaginary quaternions:

$$
I_{a}^{2}=-\mathbb{1} \quad \text { and } \quad I_{a} I_{b}=\epsilon_{a b c} I_{c} \text { when } a \neq b
$$

which are covariantly constant with respect to the metric $g$. Corresponding to these, there exist a triplet of symplectic structures (i.e. closed non-degenerate 2 -forms) $\omega_{a}(\cdot, \cdot)=$ $g\left(I_{a} \cdot, \cdot\right)$. Hyper-Kähler manifolds can be thought of complex manifolds by choosing one complex structure, say $I_{3}$, out of $I_{1,2,3}$. Let us define complex coordinates $\phi^{i}(i=1, \cdots, 2 n)$ using the chosen complex structure $I_{3}$. Then the symplectic form for $I_{3}$ can be written in the complex coordinates as $\omega_{\mathbb{R}}=i g_{i j^{*}} d \phi^{i} \wedge d \phi^{* j}$. In addition they naturally have a holomorphic symplectic structure, made of the rest of the complex structures: it is given by the $(2,0)$-form $\omega_{\mathbb{C}}=g_{i k^{*}}\left[\left(I_{1}\right)^{k^{*}}{ }_{j}+i\left(I_{2}\right)^{k^{*}}{ }_{j}\right] d \phi^{i} \wedge d \phi^{j}$.

SUSY NLSM with eight supercharges are defined by the target hyper-Kähler manifolds with the complex coordinates $\phi^{i}$ as scalar fields. In order for the walls to exist, we need a potential term in the Lagrangian lifting the vacuum degeneracy. The Lagrangian is severely restricted by high degree of SUSY, so that the bosonic part of the Lagrangian in $d$ space-time dimensions should be written as 38

$$
\mathcal{L}_{\text {boson }}=g_{i j^{*}} \partial_{\mu} \phi^{i} \partial^{\mu} \phi^{* j}+\sum_{\alpha} g_{i j^{*}} K_{\alpha}^{i} K_{\alpha}^{* j}
$$


where $K_{\alpha}^{i}(\alpha=1, \cdots, 6-d)$ generates an isometry preserving the three complex structures. Such isometries are called tri-holomorphic Killing vectors ${ }^{1}$. We absorbed the mass parameters into the Killing vectors. This NLSM with potential term is called the massive hyper-Kähler NLSM, and it can be obtained by a Sherk-Schwarz dimensional reduction [43] from a massless NLSM in six spacetime dimensions [42. In five dimensions $(d=5)$, we can choose one isometry $K^{i}$ so that the potential for the hypermultiplet can be given by the square of the single Killing vector. Although we can have options of choosing two (three, four) different Killing vectors to obtain a potential term in four (three, two) dimensions, it is enough to consider one Killing vector to construct $1 / 2$ BPS walls. Therefore we take a single tri-holomorphic Killing vector for the potential throughout this paper.

From the expression (2.2), one can see that the SUSY vacua are precisely the fixed points of the $U(1)$ action by $K^{i}$. Let us take coordinates $\phi^{i}$ so that their origin $\phi^{i}=0$ becomes a chosen fixed point. Then, we can expand the Killing vector as $K^{i}=q_{j}^{i} \phi^{j}+O\left(\phi^{2}\right)$ near each vacuum. Choosing suitable local coordinates, one can arrange so that $K^{i}=i q^{i} \phi^{i}$ with $q^{i}$ real (no summation on $i$ ). The eigenvalue $q^{i}$ gives the charge and the mass of the $i$-th scalar field.

We assume that there exist at least two vacua to have domain walls. Energy density of domain walls per unit volume perpendicular to $y$ direction can be bounded from below as follows:

$$
\begin{aligned}
\mathcal{E} & =\int d y\left(g_{i j *} \frac{\partial \phi^{i}}{\partial y} \frac{\partial \phi^{* j}}{\partial y}+g_{i j *} K^{i} K^{* j}\right) \\
& =\int d y g_{i j *}\left(\frac{\partial \phi^{i}}{\partial y}-i K^{i}\right)\left(\frac{\partial \phi^{* j}}{\partial y}+i K^{* j}\right)+i \int d y g_{i j *}\left(K^{i} \frac{\partial \phi^{* j}}{\partial y}-\frac{\partial \phi^{i}}{\partial y} K^{* j}\right) \\
& \geq i \int d y g_{i j *}\left(K^{i} \frac{\partial \phi^{* j}}{\partial y}-\frac{\partial \phi^{i}}{\partial y} K^{* j}\right)=[D]_{y=-\infty}^{y=+\infty}
\end{aligned}
$$

where $D$ is the moment map (or the Killing potential) ${ }^{2}$ for $K^{i}$ which is defined by $K^{i}=$ $-i g^{i j^{*}} \partial_{j^{*}} D$. The inequality is saturated [16] if the BPS equation

$$
\frac{\partial \phi^{i}}{\partial y}=i K^{i}
$$

is satisfied, and then the energy density is given by $[D]_{y=-\infty}^{y=+\infty}$.

Let us analyze the flow near each of the vacuum. Take suitable coordinates as before, so that $K^{i}=i q^{i} \phi^{i}$ (no summation) and $q^{i}$ be real. The BPS equation near the vacuum is

\footnotetext{
${ }^{1}$ When one couples the hyper-Kähler sigma model to $N$ vector multiplets $(I=1, \ldots, N)$ using triholomorphic isometries $K^{i, I}$, there appear additional $(6-d)$-parameters $q_{\alpha}^{I}(\alpha=1, \ldots, 6-d)$ for each vector multiplet. When we use the vector multiplets just as spurions, they give masses to hypermultiplets leading to the potential term in the Lagrangian (2.2). Things will become more interesting and complicated when one couples the system above to dynamical vector fields. That direction of research will be pursued elsewhere.

${ }^{2}$ Since the Killing vector is tri-holomorphic, the moment map is also a triplet $\vec{D}=\left(D_{1}, D_{2}, D_{3}\right)$. Using $S U(2)_{R}$ transformation, we can always take $[\vec{D}]_{y=-\infty}^{y=+\infty}$ to lie along the third direction. This choice is most convenient since we have selected $I_{3}$ as the complex structure corresponding to the four supercharges.
} 
then approximated by

$$
\frac{\partial \phi^{i}}{\partial y}=-q^{i} \phi^{i} .
$$

This means that the wall flows in or out along the $i$-th scalar field according to the sign of the $U(1)$ charge $q^{i}$. As our theories have eight supercharges, $q^{i}$ come in pairs of $+q$ and $-q$. Hence, out of the $4 n$ real dimensions of the tangent space, always $2 n$ is of the incoming direction and $2 n$ is of the outgoing direction. It is known that the BPS equation (2.4) is precisely the Morse flow with the Morse function $D$ [19]. The Morse index, which is defined as the number of outgoing directions of Morse flows, is always $2 n$ irrespective of the vacuum. Apparently, there is not much of geometrical information carried by the Morse flow at this level of generality.

Another point to be noted is that hyper-Kähler manifolds are Ricci-flat, and that compact Ricci-flat manifolds with trivial $\pi_{1}$ admit no Killing vectors. Therefore our hyperKähler manifolds should be non-compact. The Morse function $D$ diverges in general if the Morse flows are going to infinities along the non-compact directions. So these flows give infinite wall tension (2.3) and we have to discard them. We will find in the next subsection that the Morse indices will give rich information when they are calculated on a compact submanifold of the hyper-Kähler manifold.

\subsection{BPS flow near special Lagrangian submanifold}

A middle dimensional submanifold $M$ with the holomorphic symplectic form $\omega_{\mathbb{C}}$ restricted to $M$ being zero, $\left.\omega_{\mathbb{C}}\right|_{M}=0$, is called a special Lagrangian submanifold. In the following we suppose that the target hyper-Kähler manifold contains a compact special Lagrangian submanifold $M$. Here $M$ is Kähler with respect to non-vanishing $\left.\omega_{\mathbb{R}}\right|_{M}$. Then, the neighborhood of $M$ inside the hyper-Kähler manifold can be identified with $T^{*} M$. We can utilize this description to analyze the structure of the flow near $M$. The case of $M=\mathbb{C} P^{n}$ manifold has been studied along these lines in Ref. [19] using the Morse theory, and one might suppose that the case already analyzed is sufficiently generic. In this section, however, we will encounter with a lot of surprises.

Let us first recall the hyper-Kähler structure on $T^{*} M$. The manifold $T^{*} M$ has the holomorphic symplectic structure, which is the necessary conditions for any manifold to have a hyper-Kähler metric. Mathematicians have found 44] that there exists a unique hyper-Kähler metric $g$ on $T^{*} M$, which satisfies the condition:

(*) $g$ coincides with the given metric on $M$ when restricted on it, and is invariant under the $U(1)$ rotation along the cotangent direction.

There is also physical realization of these metrics using the projective superspace formalism 45. It should be noted that the metric may only be defined on some neighborhood of the zero section and that the geodesic length to infinity along the cotangent direction may be finite. This remark will be important when we study the global structure of the flow in section 3. We focus in this section in the neighborhood of the zero section. 
We decompose the complex scalars $\phi^{i}(i=1, \cdots, 2 n)$ into two sets $\left(z^{i}, \tilde{z}_{i}\right)$ so that $z^{i}$ $(i=1, \cdots, n)$ be the local complex coordinates of the base $M$ and $\tilde{z}_{i}$ be their canonical conjugates with respect to $\omega_{\mathbb{C}}$ parametrizing the cotangent direction. The holomorphic symplectic form can be written in these coordinates as $\omega_{\mathbb{C}}=\omega_{1}+i \omega_{2}=d z^{i} \wedge d \tilde{z}_{i}$. Let $k^{i}$ be a holomorphic Killing vector of $M$. The action of $k^{i}$ on $M$ naturally induces a vector field $K^{i}=\left(k^{i},-\tilde{z}_{j} \partial_{i} k^{j}\right)$ defined on all of $T^{*} M$. Let us now check that $K^{i}$ preserves the holomorphic symplectic form. Using the Lie derivatives of the one-forms

$$
\mathcal{L}_{K} d z^{i}=\partial_{j} k^{i} d z^{j} \quad \text { and } \quad \mathcal{L}_{K} d \tilde{z}_{i}=-\partial_{i} k^{j} d \tilde{z}_{j}-\tilde{z}_{j} \partial_{i} \partial_{l} k^{j} d z^{l},
$$

we have

$$
\mathcal{L}_{K} \omega_{\mathbb{C}}=\partial_{j} k^{i} d z^{j} \wedge d \tilde{z}_{i}+d z^{i} \wedge\left(-\partial_{i} k^{j} d \tilde{z}_{j}-\tilde{z}_{j} \partial_{i} \partial_{l} k^{j} d z^{l}\right)=0 .
$$

Secondly, let us show that $K^{i}$ preserves the Kähler form $\omega_{\mathbb{R}}$ on $T^{*} M$. Consider the integrated flow $g_{y}: T^{*} M \rightarrow T^{*} M$ generated by $K^{i}: d g_{y} /\left.d y\right|_{y=0}=K^{i}$. From the assumption that $k^{i}$ is a holomorphic isometry of $M$, we have $\left.g_{y}^{*}\left(\omega_{\mathbb{R}}\right)\right|_{M}=\left.\omega_{\mathbb{R}}\right|_{M}$. Additionally, the action of $K^{i}$ commutes with the $U(1)$ rotation along the cotangent direction. That is, $g_{y}^{*}\left(\omega_{\mathbb{R}}\right)$ also satisfies the above condition $(*)$. Hence, we have $g_{y}^{*}\left(\omega_{\mathbb{R}}\right)=\omega_{\mathbb{R}}$ from the uniqueness. Summarizing, we have shown that $K^{i}$ is tri-holomorphic on $T^{*} M$, so that we can use $K^{i}$ to give masses to the hypermultiplets.

The BPS equation (2.4) for walls then becomes

$$
\begin{aligned}
& \frac{d z^{i}}{d y}=i k^{i}, \\
& \frac{d \tilde{z}_{i}}{d y}=-i \tilde{z}_{j} \frac{\partial k^{j}}{\partial z^{i}}
\end{aligned}
$$

when one uses the variables $z^{i}$ and $\tilde{z}_{i}$. Hence, we can first solve the differential equation (2.8) for $z$, and then can solve the equation (2.9) for $\tilde{z}$ by plugging in the solution just obtained for $z$.

Let us consider a wall interpolating two vacua $\alpha(y \rightarrow \infty)$ and $\beta(y \rightarrow-\infty)$ inside the base $M$. Then, the wall satisfies the conditions $\tilde{z}_{i}=0$ at $y \rightarrow \pm \infty$. We can think of the study of (2.9) as the study of zero modes of the operator

$$
\mathcal{D}_{1}=\mathbb{1} \frac{d}{d y}+i \mathbb{A}
$$

acting on the functions $\tilde{z}_{i}(y)$ where $\mathbb{1}$ is the unit matrix and $\mathbb{A}_{i}{ }^{j} \equiv \partial k^{j} / \partial z^{i}$.

Let us next consider the linearized version of equation (2.8) which governs the local deformation of the wall profile in the base $M$ :

$$
\frac{d \delta z^{i}}{d y}=i \frac{\partial k^{i}}{\partial z^{j}} \delta z^{j}
$$


We set the boundary condition $\delta z^{i} \rightarrow 0$ when $y \rightarrow \pm \infty$ so that the solution should describe normalizable deformation in a fixed topological sector. Hence the number of the freedom inside the base space is given by the number of the zero modes of the operator

$$
\mathcal{D}_{2} \equiv-\mathbb{1} \frac{d}{d y}+i \mathbb{A}^{\mathrm{T}}
$$

which acts on the space of $\delta z^{j}$. Alert reader will have already recognized that the operators (2.10) and (2.12) are adjoint to each other, and that the boundary conditions placed is precisely those in the setup of the index theorem on open spaces [46, 47]. Thus we immediately obtain the relation

$$
\operatorname{dim}_{\mathbb{R}} \operatorname{Ker} \mathcal{D}_{2}-\operatorname{dim}_{\mathbb{R}} \operatorname{Ker} \mathcal{D}_{1}=n_{\beta}-n_{\alpha}
$$

where $n_{\alpha, \beta}$ is the Morse index of the flow inside $M$ at the respective vacuum. The Morse index at a vacuum is defined as the dimension of the outgoing direction of the flow. It is given explicitly as the number of positive eigenvalues of the matrix $i \mathbb{A}$ at the vacuum.

Note that the Morse index of the flow on all of $T^{*} M$ is always half of the total dimensions. Therefore the above index theorem (2.13), if we consider the flow on the entire target manifold $T^{*} M$, always gives null result trivially ${ }^{3}$. Hence we need to take the Morse index of the flow restricted inside the base.

What is the physical implication of the relation (2.13)? Recall the arguments in the previous subsection. The eigenvalues of the matrix $\mathbb{A}_{i}{ }^{j}=\partial k^{j} / \partial z^{i}$ at the vacuum control the masses of the hypermultiplets there, and these in turn control the direction of the flow. If we restrict attention to the flow inside the base space only and denote the total dimensions of the base space by $n$, the stable manifold $S(\alpha)$ flowing into the vacuum $\alpha$ is of dimension $n-n_{\alpha}$ and the unstable manifold $U(\beta)$ flowing out of the vacuum $\beta$ is of dimension $n_{\beta}$. Hence, the BPS flow to $\alpha$ from $\beta$ naïvely will form a family of dimension $n_{\beta}-n_{\alpha}$, that is, if we assume the stable and the unstable manifolds intersect transversally ${ }^{4}$. This is precisely the number appearing in the right hand side of the relation (2.13). The true dimension of the deformations of the BPS flow is, on the other hand, the first term dim $\operatorname{Ker} \mathcal{D}_{2}$ in the left hand side. These arguments combined tell us that $\operatorname{dim} \operatorname{Ker} \mathcal{D}_{1}$, which counts the freedom of deformation in the cotangent direction, measures the non-transversality of the flow!

The index theorem used above is none other than the prototypical one which counts the fermionic zero modes in the instanton background in $S^{3} \times \mathbb{R}_{y}$. In that case, $\mathcal{D}_{1(2)}$ corresponds to the Dirac operator acting on spinors of positive (negative) chirality, and $n_{\alpha, \beta}$ corresponds to the winding number of the gauge field in the vacuum at $y=\infty$ $(y=-\infty)$. The index theorem has been used also to count the dimension of BPS domain walls in Abelian gauge theories [33] and in non-Abelian gauge theories [36]. There is one

\footnotetext{
${ }^{3}$ This is a generic result applicable not only to NLSM, but also to gauged linear sigma models at finite gauge coupling, as shown in our Appendix B

${ }^{4}$ We summarize the mathematical definition surrounding transversality in Appendix A
} 
crucial difference in the application of index theorems to the wall system considered in this paper from the cases previously considered. For wall systems and instantons to date, if they are BPS (or in other words they are self-dual for the case of instantons), there is a vanishing theorem which guarantees the absence of zero modes for $\mathcal{D}_{1}$. Hence, the number of fermionic zero modes around BPS instantons was precisely proportional to the instanton number. However in our case, there is no such vanishing theorem available. This means that we can have excess zero modes even for BPS walls, and that the dimension of moduli of BPS walls may be bigger than the difference of indices of the vacua connected. We will see an explicit example shortly, and there we will also find that this is rather a generic phenomenon for BPS walls.

This generalness necessitates another comment on the non-transversality. When one considers Morse flows in general (without SUSY), one can perturb the Morse function a bit to make the flow transversal. What distinguishes our situation from such generic cases is that SUSY forces the flow to be of specific type, so that we cannot take a generic perturbation. As a result, we are forced to have the non-transversal situation ${ }^{5}$.

When the manifold $M$ is toric, that is, when it can be realized using a gauged linear sigma model with four SUSY, we can obtain $T^{*} M$ as an open subset of a toric hyperKähler manifold, that is, we can realize $T^{*} M$ as a subset of the Higgs branch of a gauged linear sigma model with eight SUSY. We can study in more detail the reason for the nontransversality of the flow in those cases. Before doing that, however, let us see some typical examples exhibiting the somewhat abstract argument above.

\subsection{Example: $T^{*} \mathbb{C} P^{2}$}

Let us first study the wall on the cotangent bundle of a projective space, $T^{*} \mathbb{C} P^{2}$. This system has been analyzed by many groups e.g. [19], and can be understood as a simple example of walls on the Grassmannians [28, 29].

$\mathbb{C} P^{2}$ can be composed from three patches $U_{i} \sim \mathbb{C}^{2}$ parametrized by $\left(v_{i}, w_{i}\right),(i=1,2,3)$ :

$$
\left(v_{1}, w_{1}\right) \equiv(v, w), \quad\left(v_{2}, w_{2}\right) \equiv(v / w, 1 / w), \quad\left(v_{3}, w_{3}\right) \equiv(1 / v, w / v) .
$$

The $U(1)^{2}$ isometry is given by the phase rotation of $v$ and $w$. Hence the fixed points, i.e. the vacua, are the origins $\alpha_{i}$ of coordinate patches [41]. We choose the $U(1)$ subgroup given by $v \rightarrow e^{i m_{1} \theta} v$ and $w \rightarrow e^{i m_{2} \theta} w$ to give masses to the hypermultiplets, where $m_{1,2}$ are taken to be real. The isometry acts, near each vacuum, as

$$
v_{i} \rightarrow e^{i m_{1}^{(i)} \theta} v_{i} \quad \text { and } \quad w_{i} \rightarrow e^{i m_{2}^{(i)} \theta} w_{i}
$$

and the BPS flow is given by

$$
v_{i} \rightarrow e^{-m_{1}^{(i)} y} v_{i} \quad \text { and } \quad w_{i} \rightarrow e^{-m_{2}^{(i)} y} w_{i}
$$

\footnotetext{
${ }^{5}$ It has been already known that the holomorphic isometry often violates the transversality, see e.g. 48.
} 
for suitable masses $m_{1,2}^{(i)}$, which are linear combinations of $m_{1}$ and $m_{2}$.

Using the discrete symmetry exchanging $\alpha_{1,2,3}$, we can assume $0<m_{2}<m_{1}$ without loss of generality. Then, the schematic structure of the flow is easily read off to be like

$$
\begin{array}{ccc}
\alpha_{1}[0] & & \\
\uparrow & \nwarrow & \\
\alpha_{2}[2] & \leftarrow \alpha_{3}[4] .
\end{array}
$$

The Morse index, which is denoted in a square bracket attached to each vacuum, is given by twice the number of outgoing arrows from that vacuum. One can easily check that there is no violation of transversality. The dimension of the moduli space of walls with no cotangent component between vacua $\alpha_{i}$ and $\alpha_{j}$ is given by twice the number of sequences of arrows connecting them.

\subsection{Another example: $T^{*} F_{n}$}

Let us next consider a massive hyper-Kähler sigma model with the target $T^{*} F_{n}$. Here, $F_{n}$ is a complex two-dimensional, real four dimensional surface which is called Hirzebruch surface and is defined as the total space of some $\mathbb{C} P^{1}$ fibration over $\mathbb{C} P^{1} . n$ is an integer describing how the $\mathbb{C} P^{1}$ is fibered on the base. We can take $n$ to be positive without loss of generality because $F_{n} \simeq F_{-n}$. An explicit description of $F_{n}$ can be given by means of four patches with coordinates $\left(v_{i}, w_{i}\right) \in U_{i} \sim \mathbb{C}^{2}, i=1,2,3,4$ :

$$
\begin{array}{llrl}
\left(v_{1}, w_{1}\right) & \equiv(v, w), & \left(v_{2}, w_{2}\right)=\left(1 / v, w / v^{n}\right), \\
\left(v_{3}, w_{3}\right) \equiv(v, 1 / w), & \left(v_{4}, w_{4}\right)=\left(1 / v, v^{n} / w\right),
\end{array}
$$

where $v$ and $1 / v$ parametrize the base $\mathbb{C} P^{1}$ and $w$ and $1 / w$ the fiber $\mathbb{C} P^{1}$. These complex surfaces are toric and can be written as a vacuum manifold of a gauged linear sigma model with four supercharges. This property will be utilized in later sections.

There is a natural isometry on $F_{n}$ with the standard metric, which is given in the first patch by

$$
v \rightarrow e^{i m_{1} \theta} v, \quad w \rightarrow e^{i m_{2} \theta} w .
$$

The action can be extended to other patches. This can be used to give masses to the hypermultiplets. The vacua are given by the fixed points of the isometry, namely the origins of the four patches $U_{i} \sim \mathbb{C}^{2}$, which are denoted by $\alpha_{i}$ respectively.

The BPS flow of the wall corresponding to (2.19) is

$$
v \rightarrow e^{-m_{1} y} v, \quad w \rightarrow e^{-m_{2} y} w
$$

Let us now consider the flow and the structure of the moduli space of walls for some specific $n, m_{1}, m_{2}$. It can be read off quite easily using the method exemplified in the last 
subsection. For $m_{2}<0<m_{1}$, the flow is schematically given by

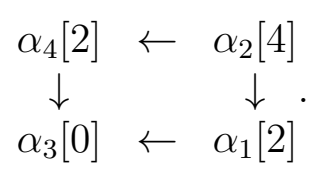

One can check that the dimensions of the BPS wall moduli in each topological sector have the naïve ones, that is, the difference of the Morse indices.

Next, let us study the case for $0<m_{2}<n m_{1}$. The flow diagram is changed to

$$
\begin{array}{ccc}
\alpha_{4}[2] & \leftarrow & \alpha_{2}[4] \\
\downarrow & & \downarrow \\
\alpha_{3}[2] & \rightarrow & \alpha_{1}[0]
\end{array} .
$$

Now the vacua $\alpha_{3}$ and $\alpha_{4}$ have the same Morse index. Naïvely, the real dimension of the moduli will be the difference of the Morse index, which is zero. At the same time the BPS wall connecting these two needs the whole $\mathbb{C} P^{1}$ to parametrize the profile inside the base space $F_{n}$. This means, from the discussion in the previous subsection, that the BPS wall connecting $\alpha_{4}$ and $\alpha_{3}$ is allowed to have non-zero expectation values of scalar fields corresponding to the cotangent direction of $T^{*} F_{n}$. The wall connecting $\alpha_{4}$ and $\alpha_{3}$ will show very peculiar dynamical behavior in this case. The analysis can be made much more concrete when one uses the gauged linear sigma model description. Thus we postpone the study of the interesting dynamics hidden in this schematic diagram to section [3.6 and let us now move on to investigate the walls in the gauged linear sigma model in general.

\section{$3 \quad$ Walls in hypertoric sigma models}

We saw in the previous sections that the wall moduli space for $T^{*} F_{n}$ is bigger than just the base space $F_{n}$, unlike the case for $T^{*} \mathbb{C} P^{n}$. Namely, there exist BPS walls which have some component along the cotangent direction, thus moving out of the base. In this section, we realize $T^{*} F_{n}$ by Abelian hyper-Kähler quotient construction [40, that is, as the Higgs branch of certain Abelian gauged linear sigma model. This viewpoint facilitates the analysis of the global structure of the BPS flow. These Abelian hyper-Kähler quotients are called the hypertoric manifolds ${ }^{6}$. Having gauged linear sigma model description, we can see more explicitly and more physically why the transversality is violated.

\footnotetext{
${ }^{6}$ These Abelian hyper-Kähler quotients are also often called toric hyper-kähler. However, these are not always toric. Recall that, in order for a manifold $M$ of complex dimension $n$ to be toric, it has to have $n$ holomorphic isometries. On the other hand, an Abelian hyper-Kähler quotient of complex dimension $2 n$ is guaranteed to have $n$ tri-holomorphic isometries. This means that it has at least $n$ holomorphic isometries, but it does not necessarily mean that they have $2 n$ of them. In fact, they are toric if and only if they are the direct product of several ALE manifolds of type A [49]. We use the word hypertoric in the rest of our paper for brevity.
} 
Let us begin with the outline of the subsections since this section is rather long. We first study in detail the Higgs branch of the massless model in subsection 3.1. We will see there that a hypertoric manifold contains several compact toric manifolds as special Lagrangian submanifolds. Then followed in subsection 3.2 we study how mass terms given through the tri-holomorphic Killing vector determine the discrete vacua. We also write down the BPS equations which govern the profile of BPS walls. In subsection 3.3 and 3.4. we will study explicit examples of such walls. These exercises tell us that the total wall moduli space are union of the compact special Lagrangian submanifolds, glued with each other at precisely the points where transversality of the flow may be violated. This is summarized in subsection 3.5. We will also see that the existence of multiple compact special Lagrangian submanifolds causes not only the possible violation of transversality, but also interesting dynamics of walls. One example is the mutual repulsion and attraction between walls treated in subsection 3.6] and another is the transmutation of a pair of walls when they encounter with each other, in subsection 3.7

\subsection{Abelian gauge theories with charged hypermultiplets}

Let us first review the construction of hypertoric manifolds using physical parlance. It is a direct extension to eight supercharges of the construction of toric manifolds using Abelian gauge theories with four supercharges [50]. The structure of these manifolds is discussed in [51] in relation to the mirror symmetry in three dimensions, and in [52, 53] in relation to brane solution in supergravity. Readers who prefer mathematical exposition can consult 49].

Let us consider a $U(1)^{N}$ gauge theory with $N_{F}$ hypermultiplets with charge matrix $q_{I}^{A}$, where $I$ and $A$ are the color and flavor indices, respectively. Let us denote the field strength and the scalar field of the $I$-th multiplet by $F_{M N}^{I}$ and $\Sigma^{I}$. If we denote the $A$ th hypermultiplet with the $S U(2)_{R}$ doublet index $i$ by $H_{A}^{i}$, the gauge group acts on the hypermultiplet via

$$
\delta_{\Lambda} H_{A}^{i}=i \Lambda^{I} q_{I}^{A} H_{A}^{i}
$$

where $\Lambda^{I}$ is the gauge parameter for the $I$-th $U(1)$. The bosonic part of the Lagrangian is then

$$
\mathcal{L}=-\frac{1}{4 g_{I}^{2}}\left(F_{M N}^{I}\right)^{2}+\frac{1}{2 g_{I}^{2}}\left(\partial_{M} \Sigma^{I}\right)^{2}+\left|\mathcal{D}_{M} H_{A}^{i}\right|^{2}-V
$$

where $\mathcal{D}_{M} H_{A}^{i}=\left(\partial_{M}+i W_{M}^{I} q_{I}^{A}\right) H_{A}^{i}$ is the covariant derivative. The potential $V$ is given by

$$
V=\left|\left(q_{I}^{A} \Sigma^{I}-m^{A}\right) H_{A}^{i}\right|^{2}+\frac{1}{2 g_{I}^{2}}\left(Y^{I a}\right)^{2},
$$

where $m^{A} \in \mathbb{R}$ is the mass of the $A$-th hypermultiplet and the auxiliary fields $Y^{I a}$ are given by their equations of motion to be

$$
Y^{I a}=g_{I}^{2}\left(c^{I a}-\sum_{A}\left(H_{A}^{i}\right)^{\dagger}\left(\sigma^{a}\right)^{i}{ }_{j} q_{I}^{A} H_{A}^{j}\right) .
$$


In this subsection we consider only the massless case, $m^{A}=0$. Let us redefine notations for hypermultiplet scalars in order to make four of supercharges apparent as

$$
H_{A}^{i=1}=h_{A}, \quad H_{A}^{i=2}=\tilde{h}^{A \dagger},
$$

where $h_{A}$ and $\tilde{h}^{A}$ are chiral fields. It is useful to define the following quantities in examining wall solutions and their properties:

$$
\mu_{A} \equiv h^{A \dagger} h_{A}-\tilde{h}^{A} \tilde{h}_{A}^{\dagger}, \quad \nu_{A} \equiv \tilde{h}^{A} h_{A}
$$

where index $A$ is not summed. By using these notations, the flatness conditions determining supersymmetric vacua are

$$
\begin{aligned}
q_{J}^{A} \Sigma^{I} h_{A} & =0 \\
q_{J}^{A} \Sigma^{I} \tilde{h}^{A} & =0 \\
q_{I}^{A} \mu_{A} & =c_{I}, \\
q_{I}^{A} \nu_{A} & =0 .
\end{aligned}
$$

Here we have taken the Fayet-Iliopoulos (FI) parameters all parallel in $S U(2)_{R}$ space. We leave the discussion for non-parallel cases to later works.

Let us concentrate on the Higgs branch, i.e. we take $\Sigma^{I}=0$. This condition is obtained automatically once one considers a generic combination of FI parameters $c_{I}$. Then the imposed conditions are

$$
q_{I}^{A} \mu_{A}=c_{I}, \quad \text { and } \quad q_{I}^{A} \nu_{A}=0
$$

The vacuum manifold $V$ is obtained by dividing this by the action of the gauge group (3.1). $V$ is a hypertoric manifold obtained by the Abelian hyper-Kähler quotient.

Next let us consider the relation to toric manifolds. First, recall that the term toric is just a fancy mathematical way of saying that it is a vacuum manifold of Abelian gauged linear sigma model with four supersymmetries. Let $K \simeq \mathbb{R}^{N_{F}-N}$ be the space spanned by $\mu_{A}$ 's modulo the constraint (3.9). The hyperplane $\mu_{A}=0$ divides $K$ to half-spaces $\mathcal{H}_{A}^{+(-)}$according to the sign of $\mu_{A}$ as illustrated in Fig. 1. A small arrow is attached to the hyperplane in the figure to indicate its orientation. These hyperplanes divide $K$ to cells $K_{a}, a=1, \ldots, m$. Let us enumerate them so that $a=1, \ldots, n$ corresponds to bounded cells and $a=n+1, \ldots, m$ unbounded ones. An example with $N_{F}=4, N=2$ is shown in Fig. 2.

For each cell $K_{a}$, let us define new variables $\left(\phi_{A}, \tilde{\phi}^{A}\right)$ based on the relative position of the hyperplane $\mu_{A}$ and $K_{a}$ :

$$
\begin{array}{lll}
\hat{q}_{I}^{A} \equiv+q_{I}^{A}, & \left(\phi_{A}, \tilde{\phi}^{A}\right) \equiv\left(h_{A}, \tilde{h}^{A}\right) & \text { if } \quad \mathcal{H}_{A}^{+} \cap K_{a} \neq \emptyset, \\
\hat{q}_{I}^{A} \equiv-q_{I}^{A}, & \left(\phi_{A}, \tilde{\phi}^{A}\right) \equiv\left(\tilde{h}^{A}, h_{A}\right) & \text { if } \quad \mathcal{H}_{A}^{-} \cap K_{a} \neq \emptyset .
\end{array}
$$




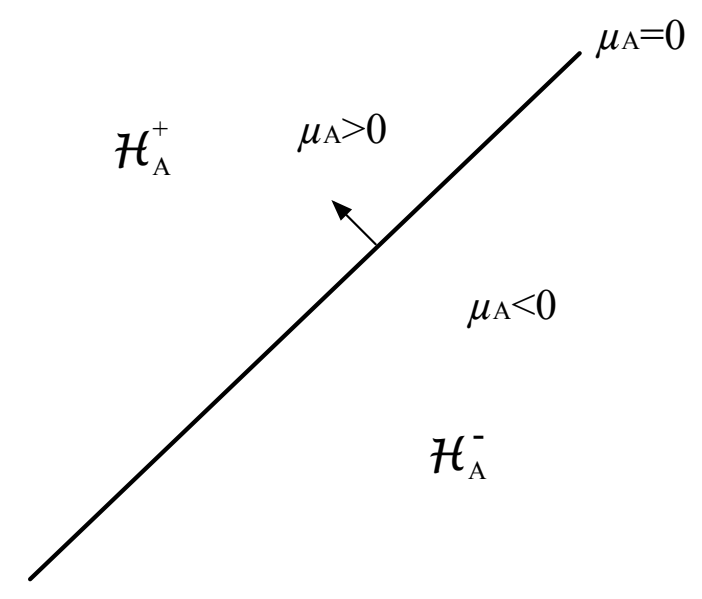

Figure 1: The definition of $\mathcal{H}_{\mathcal{A}}^{ \pm}$. The arrow indicates in which side $\mu_{A}$ is positive.

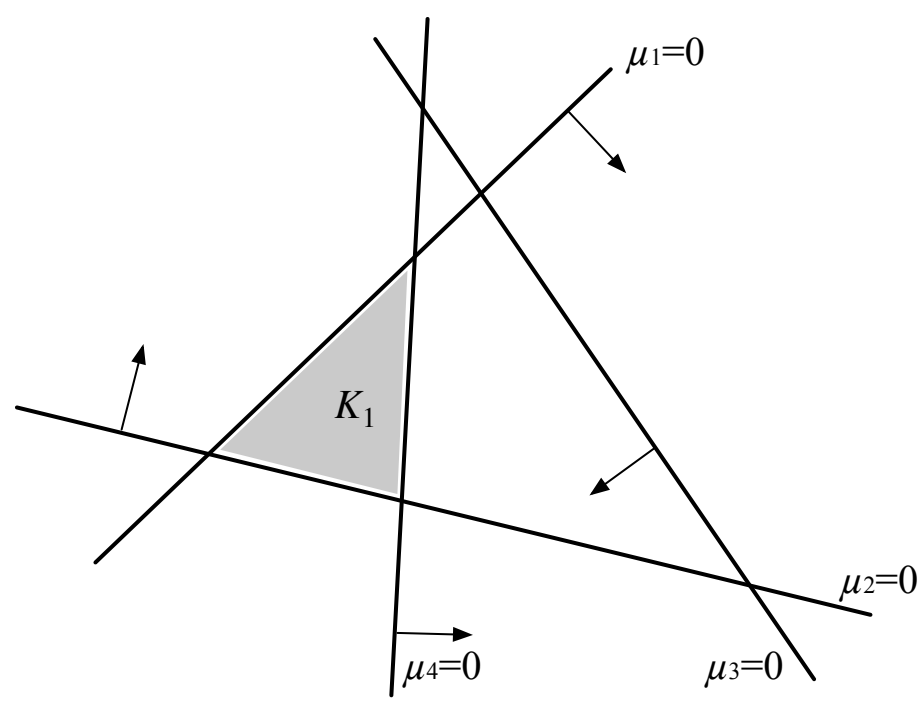

Figure 2: An example with $N_{F}=4, N=2$. 
An illustrative example is depicted in Fig. 22 The shaded region $K$ intersects with $\mathcal{H}^{+}{ }_{1}$, $\mathcal{H}^{+}{ }_{2}, \mathcal{H}^{+}{ }_{3}$ and $\mathcal{H}^{-}{ }_{4}$, thus, $\phi_{A}=\left(h_{1}, h_{2}, h_{3}, \tilde{h}^{4 \dagger}\right)$.

Consider a subspace $M_{a}$ of the hypertoric $V$ defined by $\tilde{\phi}_{A}=0$. Together with the vacuum conditions (3.11), the equation reduces to

$$
\sum_{A} \hat{q}_{I}^{A} \phi_{A} \phi_{A}^{\dagger}=c_{I}
$$

and the gauge equivalence is defined by

$$
\phi_{A} \sim \exp \left(i \Lambda^{I} \hat{q}_{I}^{A}\right) \phi_{A} \quad(\text { no summation on } A \text { ). }
$$

Each subspace $M_{a}$ is a toric manifold defined by the charge matrix $\hat{q}_{I}^{A}$ and the FI parameters $c_{I}$.

Let us determine how $M_{a}$ is embedded inside $V$. Consider a point $p=\left\langle\phi_{A}\right\rangle \in M_{a}$. By expanding (3.11) to the leading order, the neighborhood of $M_{a}$ near $p$ is determined by the equations

$$
\begin{aligned}
\operatorname{Re} \sum_{A} \hat{q}_{I}^{A} \delta \phi_{A}\left\langle\phi_{A}^{\dagger}\right\rangle & =0, \\
\sum_{A} \hat{q}_{I}^{A} \delta \tilde{\phi}^{A}\left\langle\phi_{A}\right\rangle & =0,
\end{aligned}
$$

modulo the gauge variation

$$
\delta \phi_{A} \sim \delta \phi_{A}+i \delta \Lambda^{I} \hat{q}_{I}^{A}\left\langle\phi_{A}\right\rangle \quad \text { (no summation on } A \text { ). }
$$

Gauge fixing can be naturally attained by extending (3.16) to

$$
\sum_{A} \hat{q}_{I}^{A} \delta \phi_{A}\left\langle\phi_{A}^{\dagger}\right\rangle=0
$$

Eqs. (3.19) by definition determine the tangent space $\left.T M_{a}\right|_{p}$ of the manifold $M_{a}$ at $\left\langle\phi_{A}\right\rangle$. Comparing (3.17) and (3.19), the infinitesimal displacement along $\tilde{\phi}^{A}$ direction can be naturally identified with $\left.\left.\overline{T M}_{a}\right|_{p} \simeq T^{*} M_{a}\right|_{p}$. As an easy corollary, $M_{a}$ is a special Lagrangian subspace of $V$. Summarizing, $V$ contains $T^{*} M_{a}$ for $a=1, \ldots, m$ as subsets. $M_{a}$ is compact or noncompact if $K_{a}$ is bounded or unbounded, respectively. The union of $M_{a}$ 's which is compact is called the core of $V$.

Before going to the analysis of massive models on hypertoric, let us study a bit more on the geometry of these manifolds. Consider a hypertoric $V$ of real dimension $4 n$ and notice that $\mu_{A}$ and $\nu_{A}$ modulo relations (3.11) provide real $3 n$ functions to be used as coordinates of $V$, which are invariant by the hypertoric isometries. Furthermore, points on $V$ with the same values for $\left(\mu_{A}, \nu_{A}\right)$ form a real $n$ dimensional submanifold. Thus, it is isomorphic to $T^{n}$. Summarizing, the hypertoric $V$ can be visualized as a family of $n$ dimensional tori $T^{n}$ parametrized by triplet of moment maps $\left(\mu_{A}, \nu_{A}\right)$. The subspace $\nu_{A}=0$ is precisely the union of all $M_{a}$, regardless of whether it is noncompact or not. 


\subsection{Massive hypermultiplets}

Now let us turn on hypermultiplet masses $m^{A}$ to lift the continuous degeneracy of vacua, so that we can consider walls interpolating the discrete vacua. For simplicity and to extract essence, we assume that the FI parameters $c_{I}$ are sufficiently generic so that no Coulomb branch remains. Then, we arrange the masses $m^{A}$ so that no hypermultiplet remains massless. The vacua are now isolated and the equations determining those vacua are

$$
\begin{aligned}
\left(q_{I}^{A} \Sigma^{I}-m^{A}\right) h_{A} & =0, \\
\left(q_{I}^{A} \Sigma^{I}-m^{A}\right) \tilde{h}^{A} & =0, \\
q_{I}^{A} \mu_{A} & =c_{I}, \\
q_{I}^{A} \nu_{A} & =0 .
\end{aligned}
$$

Let us determine vacua by solving these conditions. If we consider $N_{\mathrm{F}}$ equations $q_{I}^{A} \Sigma^{I}-$ $m^{A}=0$ which define hyperplanes $\sigma^{A}$, only $N$ equations within them can be solved at once with the generic $m^{A}$. Thus the first two equations (3.20) and (3.21) imply that $\left(N_{\mathrm{F}}-N\right)$ hypermultiplets must vanish. Let $\alpha$ be a set of $N$ flavor indices selected among $N_{\mathrm{F}}$ flavor indices, that is, $\alpha \equiv\left\{A_{1} A_{2} \cdots A_{N}\right\}$. Furthermore, let $\left(q_{\alpha}\right)_{I}^{A_{J}}$ be a $N \times N$ submatrix of $q_{I}^{A}$ with $\left\{A_{J}\right\} \in \alpha$. If $q_{\alpha}$ is invertible, i.e. $\operatorname{det}\left(q_{\alpha}\right)_{I}^{A_{J}} \neq 0$, the equations for the supersymmetric vacuum can be solved as follows:

$$
\begin{aligned}
\left\langle\Sigma^{I}\right\rangle_{\alpha} & =\left(q_{\alpha}^{-1}\right)_{I}^{A_{J}} m_{A_{J}}, \\
\left\langle\mu_{A_{I}}\right\rangle_{\alpha} & =\left(q_{\alpha}^{-1}\right)_{J}^{A_{I}} c^{J}, \\
\left\langle\nu_{A_{I}}\right\rangle_{\alpha} & =0
\end{aligned}
$$

and we set that all $\left(h_{A}, \tilde{h}^{A}\right)$ vanish for $\{A\} \notin \alpha$. The assumptions of generic $c_{I}$ and $m^{A}$ imply that there are no other kinds of solutions. The condition $\operatorname{det}\left(q_{\alpha}\right) \neq 0$ indicates that each plane within $N$ hyperplanes defined by $\sigma^{A_{I}}$ intersects with all the other $(N-1)$ hyperplanes. Therefore, the above isolated vacua labeled by $\alpha$ are given by the intersecting points of $N$ hyperplanes in the space of $\Sigma^{I}$ 's. The last equation means that either $h_{A}$ or $\tilde{h}^{A}$ must be zero for each flavor $A$.

These vacua can be also determined from the viewpoint of section 2. The set of $m^{A}$ determines a $U(1)$ subgroup of $U(1)^{N_{F}}$ flavor symmetry, and this descends to a triholomorphic isometry on the hypertoric manifold. Each vacuum corresponds to the fixed point of the isometry. For generic $m^{A}$, this fixed point should become a fixed point for all the $U(1)^{N_{F}}$ symmetry. As the hyperplane $\mu_{A}=0$ is precisely the fixed points of $A$-th $U(1)$ subgroup of $U(1)^{N_{F}}$, each vacuum $\alpha$ should be on the intersection of $\left(N_{F}-N\right)$-hyperplanes $\mu_{A}=0(A \notin \alpha)$ in the space $K$. Consider the vacuum expectation values $\left(\left\langle h_{A}\right\rangle,\left\langle\tilde{h}^{A}\right\rangle\right)$. They are invariant under $A$-th $U(1)$ for $A \notin \alpha$. They are, however, not invariant by the $B$-th $U(1)$ for $B \in \alpha$, but this can be gauged away using the $U(1)^{N}$ symmetry. In this sense, the vacuum is labeled by the locking of flavor and color symmetry.

Let us now move on to study a wall interpolating two vacua labeled by $\alpha=\left\{A_{1}, A_{2}, \ldots, A_{N}\right\}$ and $\beta=\left\{B_{1}, B_{2}, \ldots, B_{N}\right\}$ respectively. We would like to determine BPS equations and 
the wall tension by performing the Bogomol'nyi completion of wall energy density under the wall ansatz. The energy density is given by

$$
\begin{aligned}
\mathcal{E}= & \frac{1}{2 g_{I}^{2}}\left(\partial_{y} \Sigma^{I}-g_{I}^{2}\left(c_{I}-q_{I}^{A} \mu_{A}\right)\right)^{2}+\frac{1}{2 g_{I}^{2}}\left|g_{I}^{2} q_{I}^{A} \nu_{A}\right|^{2} \\
& +\left|\mathcal{D}_{y} h_{A}+\left(q_{I}^{A} \Sigma^{I}-m^{A}\right) h_{A}\right|^{2}+\left|\mathcal{D}_{y} \tilde{h}^{A}-\left(q_{I}^{A} \Sigma^{I}-m^{A}\right) \tilde{h}^{A}\right|^{2} \\
& +\partial_{y}\left(c^{I} \Sigma^{I}-q_{I}^{A} \Sigma^{I} \mu_{A}+m^{A} \mu_{A}\right) .
\end{aligned}
$$

The topological charge can be read from the last term to give

$$
T_{\alpha \leftarrow \beta}=\int_{-\infty}^{+\infty} d y \partial_{y} f, \quad \text { with } \quad f \equiv c^{I} \Sigma^{I}-q_{I}^{A} \Sigma^{I} \mu_{A}+m^{A} \mu_{A}
$$

The BPS equations are obtained from Eq. (3.27) as

$$
\begin{aligned}
\partial_{y} \Sigma^{I} & =g_{I}^{2}\left(c^{I}-q_{I}^{A} \mu_{A}\right), \\
-2 g_{I}^{2} q_{I}^{A} \nu_{A} & =0 \\
\left(\partial_{y}+q_{I}^{A}\left(\Sigma^{I}+i W_{y}^{I}\right)\right) h_{A} & =h_{A} m^{A} \\
\left(\partial_{y}-q_{I}^{A}\left(\Sigma^{I}+i W_{y}^{I}\right)\right) \tilde{h}^{A} & =-\tilde{h}^{A} m^{A} .
\end{aligned}
$$

When this equations are satisfied, the energy density becomes equal to $[f]_{y=-\infty}^{y=+\infty}$.

Formal solutions can be found by using the method which we have developed in the previous works [20, 28, 29]: First, we define functions $\psi^{I}(y)$ by the relation

$$
\Sigma^{I}(y)+i W_{y}^{I}(y)=\partial_{y} \psi^{I}(y) .
$$

Let us note that $\psi^{I}$ is defined up to an additive constant. The hypermultiplets can be expressed using $\psi^{I}$ by solving (3.30), (3.31), and (3.32):

$$
\begin{aligned}
h_{A}(y) & =h_{0 A} e^{-q_{I}^{A} \psi^{I}+m^{A} y}, \\
\tilde{h}^{A}(y) & =\tilde{h}_{0}^{A} e^{+q_{I}^{A} \psi^{I}-m^{A} y} \\
\nu_{A} & =\tilde{h}_{0}^{A} h_{0 A}=0 .
\end{aligned}
$$

Here, $h_{0 A}$ and $\tilde{h}_{0}^{A}$ are vectors with $N_{F}$ elements. We call these vectors as moduli matrices in the rest of the paper, following the terminology in Refs. 28, 29]. Eq. (3.36) follows from the boundary conditions $\nu_{A}=0$ at $y \rightarrow \pm \infty$. Hence, either $h_{A}$ or $\tilde{h}^{A}$ must vanish identically for each flavor $A$. Thus we find that $\mu_{A}$ can not flip the sign in a wall configuration. This fact means that there is no BPS walls which interpolate from one vacuum with non-vanishing vacuum expectation values (VEV) of $h_{A}$ to another vacuum with non-vanishing VEV of $\tilde{h}^{A}$. That is, BPS walls cannot cross hyperplanes $\mu_{A}=0$. Based on these considerations, we can identify sets of vacua which can be interpolated with each other by wall solutions. 
Finally, $\psi$ itself is solved by plugging (3.33) into (3.29):

$$
\begin{aligned}
\frac{1}{g_{I}^{2}} \partial_{y}^{2} \operatorname{Re}\left(\psi^{I}\right) & =c^{I}-q_{I}^{A} \mu_{A}(y) \\
& =c^{I}-q_{I}^{A}\left(\left|h_{0 A}\right|^{2} e^{-2 q_{J}^{A} \operatorname{Re}\left(\psi^{J}\right)+2 m^{A} y}-\left|\tilde{h}_{0}^{A}\right|^{2} e^{+2 q_{J}^{A} \operatorname{Re}\left(\psi^{J}\right)-2 m^{A} y}\right) .
\end{aligned}
$$

Once this second order nonlinear differential equation (3.37) is solved for given moduli matrices $h_{0 A}$ and $\tilde{h}_{0}^{A}$, all fields can be determined from Eqs. (3.34)-(3.36). Eqs. (3.34) and (3.35) show that the additive ambiguity of $\psi^{I}$ results in the $G L(1, \mathbb{C})^{N}$ equivalence on the moduli matrices

$$
\left(h_{0 A}, \tilde{h}_{0}^{A}\right) \simeq\left(\left(\lambda_{I}\right)^{q_{I}^{A}} h_{0 A},\left(\lambda_{I}\right)^{-q_{I}^{A}} \tilde{h}_{0}^{A}\right), \quad \lambda_{I} \in \mathbb{C}^{*} \equiv \mathbb{C}-\{0\} .
$$

Thus the equivalence class of the moduli matrices defined by Eq. (3.38) describes the moduli spaces of walls, which are submanifolds of the hyper-Kähler manifold $V$. Note that we eventually take the strong gauge coupling limit $g_{I} \rightarrow \infty$ since we are now interested only in the Higgs branch which we constructed using the hyper-Kähler quotient. Therefore the differential equation (3.37) reduces to mere algebraic equations

$$
c_{I}=q_{I}^{A} \mu_{A}(y)
$$

throughout the wall profile. Combined with a trivial equation $0=q_{I}^{A} \nu_{A}(y)$, flows defined by the BPS equations can be interpreted as Morse flows on the hyper-Kähler manifold in the strong coupling limit. The Morse function for those flows is the function $f$ in the Bogomol'nyi completion (3.28)

$$
f=c^{I} \Sigma^{I}-\left(q_{I}^{A} \Sigma^{I}-m^{A}\right) \mu_{A}=m^{A} \mu_{A} .
$$

In the following, we will mainly treat cases in the strong coupling limit for simplicity.

\subsection{Walls in gauged linear sigma model of the form $T^{*} \mathbb{C} P^{2}$}

Let us first study the hypertoric realization of $T^{*} \mathbb{C} P^{2}$. $\mathbb{C} P^{2}$ is specified as a toric manifold by the charge matrix

$$
q_{I}^{A}=(1,1,1) .
$$

The hypertoric manifold $V$ is determined by the same charge matrix. The arrangement of three hyperplanes and the computed flows are depicted in Fig. 3. From this we can see that $V$ contains $\mathbb{C} P^{2}$ as the only compact Kähler special Lagrangian submanifold and $V=T^{*} \mathbb{C} P^{2}$. Furthermore, all the vacua of the massive $T^{*} \mathbb{C} P^{2}$ model reside on $\mathbb{C} P^{2}$. Hence, nothing strange happens. The moduli space for walls in this case are given by the homogeneous coordinates $h_{0 A}$ with $\tilde{h}_{0}^{A}=0$ under the identification

$$
\left(h_{01}, h_{02}, h_{03}\right) \simeq \lambda\left(h_{01}, h_{02}, h_{03}\right),
$$



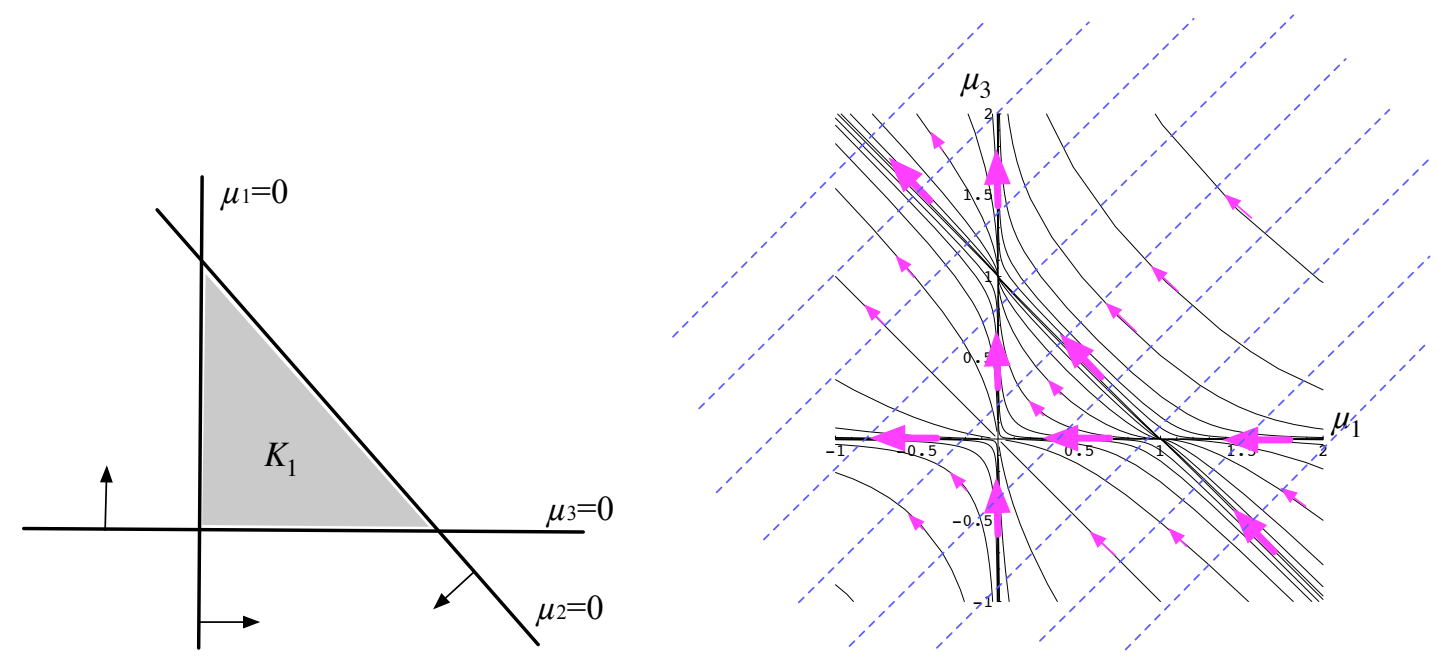

Figure 3: Left: Hyperplane arrangement for $T^{*} \mathbb{C} P^{2}$; Right: Flows in $T^{*} \mathbb{C} P^{2}$ with masses $m^{A}=(1,0,-1)$. Dashed (blue) lines are the level sets of the Morse function $m^{A} \mu_{A}$.

which describe $\mathbb{C} P^{2}$. All flows satisfy the transversality. We can chase the flow starting from a point outside the base $\mathbb{C} P^{2}$. They all go to infinity in the field space, however, as $y$ goes to $\pm \infty$ as one can see. Therefore they do not correspond to walls interpolating between two vacua. ${ }^{7}$ What will happen then, when there are vacua outside the original base toric manifold? We will see this in the next subsection and how this is related to the violation of transversality.

\subsection{Walls in the gauged linear sigma model containing $F_{n}$}

Let us next examine the hypertoric manifold containing $T^{*} F_{n}$. This model admits walls which flow into cotangent directions. A charge assignment matrix giving this manifold by the quotient construction is as follows:

$$
q_{I}^{A}=\left(\begin{array}{cccc}
1 & 1 & 0 & 0 \\
0 & -n & 1 & 1
\end{array}\right) .
$$

Using this charge matrix, we can write down concretely the flatness conditions (3.20)-(3.23) for SUSY vacua

$$
\begin{aligned}
\left(\Sigma^{1}-m_{1}\right) h_{A} & =0, & \left(\Sigma^{1}-n \Sigma^{2}-m_{2}\right) h_{A} & =0, \\
\left(\Sigma^{2}-m_{3}\right) h_{A} & =0, & \left(\Sigma^{2}-m_{4}\right) h_{A} & =0,
\end{aligned}
$$

together with the same equations for $\tilde{h}_{A}$, and

$$
\begin{aligned}
\mu_{1}+\mu_{2} & =c_{1}, & -n \mu_{2}+\mu_{3}+\mu_{4} & =c_{2}, \\
\nu_{1}+\nu_{2} & =0, & -n \nu_{2}+\nu_{3}+\nu_{4} & =0 .
\end{aligned}
$$

\footnotetext{
${ }^{7}$ This statement has been proved in Appendix C of Ref. 29] for more general case of $V=T^{*} G r_{N_{\mathrm{F}}, N_{\mathrm{C}}}$ without taking strong coupling limit.
} 


\begin{tabular}{|c||c|c||c|c|c|c|}
\hline vacua & $\Sigma^{1}$ & $\Sigma^{2}$ & $\mu_{1}$ & $\mu_{2}$ & $\mu_{3}$ & $\mu_{4}$ \\
\hline \hline$\alpha_{1}$ & $m_{2}+n m_{3}$ & $m_{3}$ & 0 & $c_{1}$ & $c_{2}+n c_{1}$ & 0 \\
$\alpha_{2}$ & $m_{2}+n m_{4}$ & $m_{4}$ & 0 & $c_{1}$ & 0 & $c_{2}+n c_{1}$ \\
$\alpha_{3}$ & $m_{1}$ & $m_{3}$ & $c_{1}$ & 0 & $c_{2}$ & 0 \\
$\alpha_{4}$ & $m_{1}$ & $m_{4}$ & $c_{1}$ & 0 & 0 & $c_{2}$ \\
$\alpha_{5}$ & $m_{1}$ & $\left(m_{1}-m_{2}\right) / n$ & $c_{1}+c_{2} / n$ & $-c_{2} / n$ & 0 & 0 \\
\hline
\end{tabular}

Table 1: The vacua of massive sigma models which contains $F_{n}$ in the sigma model target.

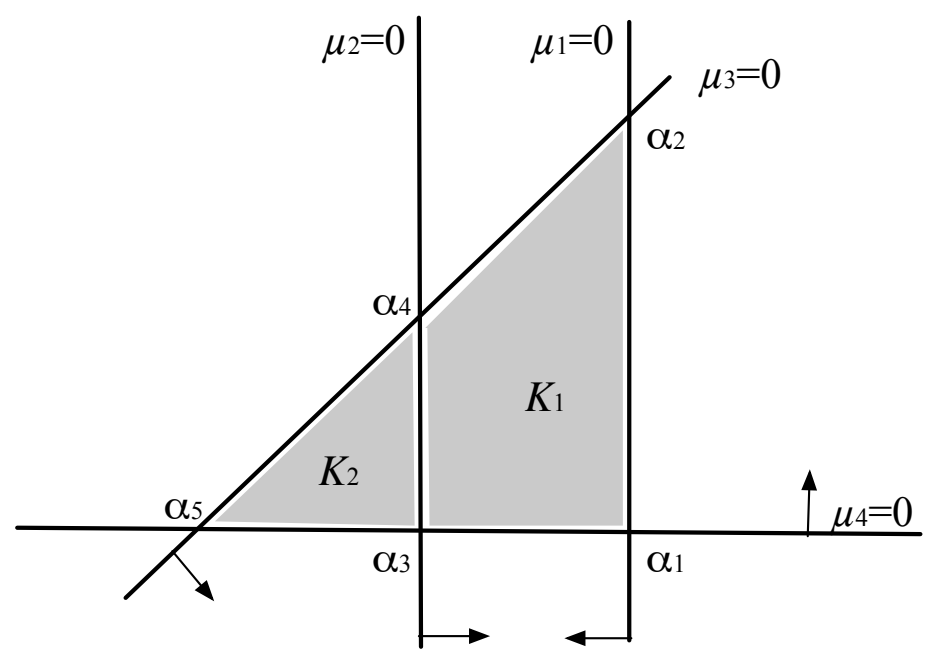

Figure 4: Hyperplane arrangement for the hypertoric which contains $T^{*} F_{n}$.

Isolated SUSY vacua are determined by these equations, which have five independent solutions. We present these solutions, which are labeled from $\alpha_{1}$ to $\alpha_{5}$, in Table 1 .

Note that, for any generic choice of the FI parameters, there exists at least one vacuum where both $h_{A}$ and $\tilde{h}^{A}$ have non-zero VEVs. For simplicity, let us choose the sign of two FI parameters to be positive from now on. Then, only the vacuum $\alpha_{5}$ has the non-zero VEV of $\tilde{h}^{2}$. We show the corresponding hyperplane arrangement and the placement of vacua in Fig. 4. As depicted there, there are two compact toric submanifold $M_{1}$ and $M_{2}$ corresponding to the bounded cells $K_{1}$ and $K_{2}$. $M_{1}$ contains vacua $\alpha_{1,2,3,4}$ and $M_{2}$ contains $\alpha_{3,4,5}$. In the former $\tilde{h}^{A}$ vanish identically but in the latter this is not the case. Since signs of $\mu_{A}$ 's can not change in the wall configuration, BPS walls cannot interpolate between the vacuum $\alpha_{5}$ and $\alpha_{1,2}$. The total moduli space of BPS walls of this model is the union of $M_{1}$ and $M_{2}$. The intersection of the two $M_{1} \cap M_{2}$ is complex one dimensional.

Homogeneous and inhomogeneous coordinate representations of $M_{a}(a=1,2)$, can be tabulated as follows: Firstly, for $a=1$, homogeneous coordinates describing $M_{1}$ are given 
by

$$
M_{1}:\left(h_{01}, h_{02}, h_{03}, h_{04}\right), \text { others }=0,
$$

with the $G L(1, \mathbb{C})^{2}$ symmetry

$$
\left(h_{01}, h_{02}, h_{03}, h_{04}\right) \simeq\left(\lambda_{1} h_{01}, \lambda_{1} h_{02}, h_{03}, h_{04}\right) \simeq\left(h_{01}, \lambda_{2}^{-n} h_{02}, \lambda_{2} h_{03}, \lambda_{2} h_{04}\right) .
$$

Therefore, the coordinate patches with inhomogeneous coordinates $(v, w)$ can be determined as follows:

\begin{tabular}{|c||c|c|c|c|}
\hline patch & $h_{01}$ & $h_{02}$ & $h_{03}$ & $h_{04}$ \\
\hline$U_{1}$ & $w$ & 1 & 1 & $v$ \\
$U_{2}$ & $w / v^{n}$ & 1 & $1 / v$ & 1 \\
$U_{3}$ & 1 & $1 / w$ & 1 & $v$ \\
$U_{4}$ & 1 & $v^{n} / w$ & $1 / v$ & 1 \\
\hline
\end{tabular}

We can see that the transition functions for $M_{1}$ are precisely the ones for $F_{n}$ as described in section 2.4 .

Secondly, let us analyze the case $a=2$ : homogeneous coordinates are given by exchanging $h_{02}$ for $\tilde{h}_{0}^{2}$ as

$$
M_{2}:\left(h_{01}, \tilde{h}_{0}^{2}, h_{03}, h_{04}\right), \text { others }=0 .
$$

with the $G L(1, \mathbb{C})^{2}$ symmetry,

$$
\left(h_{01}, \tilde{h}_{0}^{2}, h_{03}, h_{04}\right) \simeq\left(\lambda_{1} h_{01}, \lambda_{1}^{-1} \tilde{h}_{0}^{2}, h_{03}, h_{04}\right) \simeq\left(h_{01}, \lambda_{2}^{n} \tilde{h}_{0}^{2}, \lambda_{2} h_{03}, \lambda_{2} h_{04}\right) .
$$

In this manifold $h_{01}$ always takes non-zero values because of $\mu_{1}>0$ and can be fixed to one by the first symmetry shown above. Then the second symmetry defines $M_{2}=W \mathbb{C} P_{1,1, n}^{2}$, which is described by three coordinate patches with inhomogeneous coordinates $(u, v)$ as follows:

\begin{tabular}{|c||c|c|c|c|}
\hline patch & $h_{01}$ & $\hat{h}_{0}^{2}$ & $h_{03}$ & $h_{04}$ \\
\hline$\widetilde{U}_{3}$ & 1 & $1 / u^{n}$ & 1 & $v$ \\
$\widetilde{U}_{4}$ & 1 & $1 /(u v)^{n}$ & $1 / v$ & 1 \\
$\widetilde{U}_{5}$ & 1 & 1 & $u$ & $u v$ \\
\hline
\end{tabular}

Next, let us concentrate on the intersection of two submanifolds where $\mu_{2}=0$, that is, $w \rightarrow \infty$ in $M_{1}$ and $u \rightarrow \infty$ in $M_{2}$, and we find

$$
\left(h_{01}, 0, h_{03}, h_{04}\right) \simeq(1,0,1, v) \simeq(1,0,1 / v, 1) .
$$

It is thus clear that $M_{1} \cap M_{2}=\mathbb{C} P^{1}$ and the vacua $\alpha_{3}$ and $\alpha_{4}$ are on that intersection. 
Therefore, we conclude that the total moduli space of walls in this model is the union of special Lagrangian submanifolds $F_{n}$ and $W \mathbb{C} P^{2}{ }_{1,1, n}$ with $\mathbb{C} P^{1}$ as their intersection.

Now let us write down wall solutions explicitly and investigate directions of flows. By changing mass parameters, flow directions and indices at each vacuum change. Types of flow can be classified into the following three cases:

Case I; $m_{3}>m_{4}, m_{2}+n m_{3}>m_{1}>m_{2}+n m_{4}$,

Case II ; $m_{3}>m_{4}, m_{1}>m_{2}+n m_{3}$,

Case III ; $m_{3}>m_{4}, m_{2}+n m_{4}>m_{1}$,

and negative thereof.

\subsubsection{Case I}

In the case I, $m_{3}>m_{4}, m_{2}+n m_{3}>m_{1}>m_{2}+n m_{4}$, the flow structure is as follows

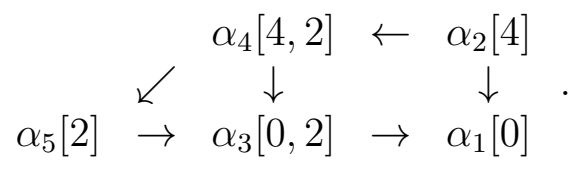

We calculated the Morse indices of the flow inside $M_{1}$ and $M_{2}$ and showed the results in the square brackets after the symbol designating the vacua. For $\alpha_{3}$ and $\alpha_{4}$ which are contained in both $M_{1}$ and $M_{2}$, the second numbers in the square brackets denote Morse indices on $M_{1}=F_{n}$, and the first numbers in them are for $M_{2}=W \mathbb{C} P_{1,1, n}^{2}$. The wall connecting $\alpha_{4}$ and $\alpha_{3}$ breaks transversality from the viewpoint of $F_{n}$, because two vacua $\alpha_{4}$ and $\alpha_{3}$ have the same Morse index. From the index theorem in Eq. (2.13), there should be BPS walls which flow out along the cotangent direction from $\alpha_{4}$ and come back from that direction to $\alpha_{3}$. This is precisely the walls depicted in the left half of the diagram (3.51).

Let us explicitly work out walls interpolating between vacua $\alpha_{2}$ and $\alpha_{1}$. For simplicity, let us set $\left(m_{1}, m_{2}, m_{3}, m_{4}\right)=(0,0, m,-m)$ and $c_{1}=c_{2}=c$. We fix the gauge for $G L(1, \mathbb{C})^{2}$ symmetry and take the moduli matrix of the topological sector $\alpha_{2} \rightarrow \alpha_{1}$ to be

$$
\left(h_{0}^{1}, h_{0}^{2}, h_{0}^{3}, h_{0}^{4}\right)=\sqrt{c}\left(a, 1, \frac{1}{\sqrt{2}} e^{-\tau}, \frac{1}{\sqrt{2}} e^{\tau}\right) .
$$

Parameters $a$ and $\tau$ are the moduli. The configuration corresponding to the above moduli matrix contains three walls; the first one flows from $\alpha_{2}$ to near $\alpha_{4}$, the second flows from near $\alpha_{4}$ to near $\alpha_{3}$ and the third from near $\alpha_{3}$ to $\alpha_{1}$. We call these walls $W_{1}, W_{2}$ and $W_{3}$ respectively as in section 2.4

Before constructing explicit wall solution $\alpha_{2} \rightarrow \alpha_{1}$, let us estimate wall positions in terms of these moduli $a, \tau$. We now assume that three walls $W_{1,2,3}$ are well separated with each other for simplicity. Then field configurations are approximately equal to the vacuum 
configuration away from wall centers, since Eq. (3.45) are close to be satisfied. From Table 1. we find that the configuration becomes far from the vacuum $\alpha_{2}$ and $\alpha_{1}\left(\alpha_{4}\right.$ and $\left.\alpha_{3}\right)$ as $\mu_{1}\left(\mu_{2}\right)$ increases. So we expect $W_{1}$ and $W_{3}$ is situated around the point where the values of $\mu_{1}$ and $\mu_{2}$ cross each other, namely $\mu_{1} \approx \mu_{2}$. To express this condition and construct the wall solution, it is useful to introduce the following functions:

$$
X(y) \equiv e^{-2 \operatorname{Re} \psi^{2}}, \quad Y(y) \equiv e^{-2 \operatorname{Re} \psi^{1}}, \quad Z(y) \equiv \cosh 2(m y-\operatorname{Re} \tau) .
$$

$\mu_{A}$ are simply expressed in terms of $X, Y$ and $Z$

$$
\mu_{1}=c|a|^{2} Y, \quad \mu_{2}=c \frac{Y}{X^{n}}, \quad \mu_{3}+\mu_{4}=c X Z
$$

Then, the condition $\mu_{1} \approx \mu_{2}$ implies $|a|^{2}=X^{-n}$. On the other hand, in the limit that $\mu_{1} \gg \mu_{2}$ we obtain $|a|^{2} \gg X^{-n} \sim Z^{n}$ by solving Eq. (3.45). Therefore wall positions for $W_{1}$ and $W_{3}$ can be roughly estimated by the condition $|a|^{2} \sim Z^{n}$, which leads to the following result

$$
y \sim y_{ \pm} \equiv \frac{1}{m} \operatorname{Re}(\tau) \pm \frac{1}{n} \log |a|, \quad \text { if }|a| \gg 1 .
$$

Similarly, the position of the wall $W_{2}$ for $\alpha_{4} \rightarrow \alpha_{3}$ turns out to be $y \sim y_{0} \equiv \operatorname{Re}(\tau) / m$ by considering the crossing of $\mu_{3}$ and $\mu_{4}$. Note that the middle wall $W_{2}$ freezes at the center of the outer two walls $W_{1}$ and $W_{3}$, since there are only two moduli for three constituent walls. This situation never occurs among walls in the case of $T^{*} \mathbb{C} P^{n}$, where all flows satisfy the transversality. So we can say that the breaking of the transversality causes this result.

Now let us construct these BPS wall solutions explicitly. As we take the strong coupling limit $g^{2} \rightarrow \infty$, we obtain the following algebraic equations from Eq. (3.39)

$$
1=Y|a|^{2}+\frac{Y}{X^{n}}, \quad 1=-n \frac{Y}{X^{n}}+X Z .
$$

Eliminating $Y$, we obtain the $n+1$-th order equation in $X$

$$
|a|^{2} Z X^{n+1}-|a|^{2} X^{n}+X Z-(n+1)=0 .
$$

For $n=1$, we can solve the above equation explicitly as

$$
\begin{aligned}
Y & =\frac{X}{|a|^{2} X+1}, \\
X & =\frac{1}{2}\left(\frac{1}{Z}-\frac{1}{|a|^{2}}\right)+\sqrt{\frac{1}{4}\left(\frac{1}{Z}+\frac{1}{|a|^{2}}\right)^{2}+\frac{1}{|a|^{2} Z}}
\end{aligned}
$$

We must plug in these results to moment maps. The final result is

$$
\mu_{2}=\frac{c}{|a|^{2} X+1}, \quad \mu_{4}=\frac{c}{2} X e^{-2(m y-\operatorname{Re} \tau)} .
$$


We used two linearly independent ones from $\mu_{1,2,3,4}$. If one lets the outer two walls going to infinities $y \rightarrow \pm \infty$, by taking the limit $|a|^{2} \rightarrow \infty$, one finds that $X=Z^{-1}, Y=0$ and then

$$
\mu_{1}=c, \quad \mu_{2}=0, \quad \mu_{3}=c \frac{e^{-2(m y-\operatorname{Re} \tau)}}{\cosh 2(m y-\operatorname{Re} \tau)}, \quad \mu_{4}=c \frac{e^{2(m y-\operatorname{Re} \tau)}}{\cosh 2(m y-\operatorname{Re} \tau)},
$$

which is just the single-wall solution interpolating $\alpha_{3}$ and $\alpha_{4}$ in the case of $\mathbb{C} P^{1}$.

Next, let us study the walls connecting $\alpha_{4}$ and $\alpha_{3}$ through $M_{2}$. We express the moduli matrix using $\tau$ as

$$
\left(h_{0}^{1}, \tilde{h}_{0}^{2}, h_{0}^{3}, h_{0}^{4}\right)=\sqrt{c}\left(b, 1, \frac{1}{\sqrt{2}} e^{-\tau}, \frac{1}{\sqrt{2}} e^{\tau}\right)
$$

We obtain the following two algebraic equations for $X$ and $Y$ as before:

$$
c=c Y|b|^{2}-c \frac{X^{n}}{Y}, \quad c=n c \frac{X^{n}}{Y}+c X Z .
$$

Positions of the two walls are approximated by

$$
y \sim y_{ \pm} \equiv \frac{1}{m} \operatorname{Re}(\tau) \pm \frac{1}{n} \log |b|, \quad \text { if }|b| \gg 1,
$$

which result from the condition $\mu_{2} \sim \mu_{3}+\mu_{4}$. The explicit solution for $n=1$ is given by

$$
\begin{aligned}
X & =\frac{Y}{Z Y+1}, \\
Y & =\frac{1}{2}\left(\frac{1}{|b|^{2}}-\frac{1}{Z}\right)+\sqrt{\frac{1}{4}\left(\frac{1}{|b|^{2}}-\frac{1}{Z}\right)^{2}+\frac{2}{|b|^{2} Z}} .
\end{aligned}
$$

and the relation to the moment maps is

$$
\mu_{2}=\frac{-c}{Z Y+1}, \quad \mu_{4}=\frac{c}{2} X e^{-2(m y-\operatorname{Re} \tau)} .
$$

The structure of the flows is depicted in the left-most figure of Fig. 15. If one compresses the two walls into a single wall by taking $|b|^{2} \rightarrow 0$, one finds that $X=Z^{-1}$ and $Y=\infty$, reproducing the result (3.60). We thus have found that two moduli spaces intersect through $\mathbb{C} P^{1}$.

\subsubsection{Case II \& III}

In Case II $, m_{3}>m_{4}, m_{1}>m_{2}+n m_{3}$, the flow structure is as follows

$$
\begin{array}{rcccc}
\alpha_{4}[4,2] & \leftarrow & \alpha_{2}[4] \\
\alpha_{5}[0] & \swarrow & \downarrow & & \downarrow \\
& \leftarrow & \alpha_{3}[2,0] & \leftarrow & \alpha_{1}[2]
\end{array}
$$




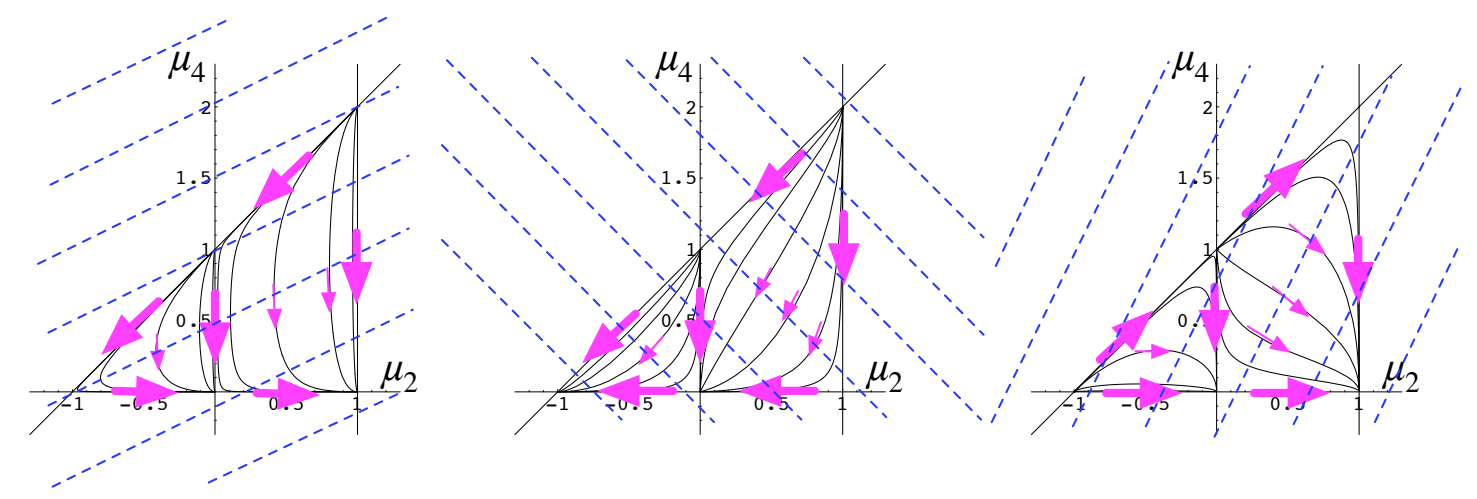

Figure 5: Numerically calculated structure of the flow in $n=1$ case. From left to the right: case I) $m^{A}=(0,0,1,-1)$; case II) $m^{A}=(1,0,0,-1)$; case III) $m^{A}=(-1,0,1,0)$. For all cases $c_{I}=(1,1)$. Dashed (blue) lines designate the contours of constant $m^{A} \mu_{A}$.

In the right half of the above diagram, the square made up of $\alpha_{1,2,3,4}$ denotes the projection of $F_{n}$ by the moment map. One can check there are no walls breaking the Morse-Smale transversality condition. From the general discussions in section 2 , there should be no walls flowing out to and coming back from the cotangent direction. Indeed, any flow from $\alpha_{4}$ with arbitrarily little cotangent component goes to the vacuum $\alpha_{5}$ and never comes back to $\alpha_{3}$.

To construct explicit wall solutions, let us take the hypermultiplet masses

$$
m^{A}=(q m, 0,0,-m)
$$

as an illustrative example. Using the symmetry (3.47), the moduli matrix can be fixed to the following form with the complex moduli parameters $\tau_{1}, \tau_{2}$

$$
\left(h_{0}^{1}, h_{0}^{2}, h_{0}^{3}, h_{0}^{4}\right)=\sqrt{c}\left(e^{-q \tau_{1}}, 1,1, e^{\tau_{2}}\right) .
$$

By a similar analysis at strong gauge coupling as in the case I, we find the wall tensions and positions as summarized in Table 2, As will be explained more in section 3.7, walls are transmuted as the relative positions are interchanged. Although the tension of individual wall changes after two walls pass through, the sum of tensions and the center of gravity are preserved. We have depicted the results in the middle of Fig. [5.

In Case III, $m_{3}>m_{4}, m_{2}+n m_{4}>m_{1}$, the flow structure is as follows

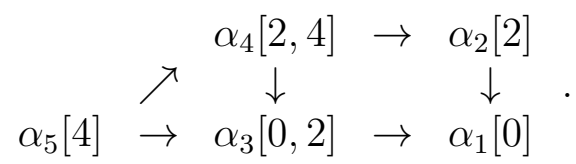

This case is very similar to Case II. The computed flow structure is given in the right-most figure of Fig. 5 , 


\begin{tabular}{|c||c|c|}
\hline wall & tension & position \\
\hline \hline $2 \rightarrow 1$ & $(1+n) c m$ & $\operatorname{Re} \tau_{2} / m$ \\
$1 \rightarrow 3$ & $q c m$ & $\operatorname{Re} \tau_{1} / m$ \\
$2 \rightarrow 4$ & $(q+n) c m$ & $\operatorname{Re} \frac{n \tau_{2}+q \tau_{1}}{(n+q) m}$ \\
$4 \rightarrow 3$ & $c m$ & $\operatorname{Re} \tau_{2} / m$ \\
\hline
\end{tabular}

Table 2: The tensions and positions of walls in case II.

\subsection{Wall moduli for generic hypertoric sigma models}

From the examples that we have studied in detail in the preceding subsections, we can understand the structure of BPS walls of the massive hypertoric sigma models. Let us recall that a hypertoric manifold contains several possibly noncompact toric manifolds $M_{a}$ as special Lagrangian submanifolds, and the Higgs branch of the vacua $V$ of the massless model is of the form

$$
V=\bigcup_{a} T^{*} M_{a}
$$

Isolated vacua of a corresponding massive theory sit on some of these $M_{a}$. Next, we know that $\nu_{A}$ is zero throughout the flow because of the BPS equation. Hence, BPS walls live in one of $M_{a}$, although $M_{a}$ may be noncompact. Solutions of BPS equation starting from such non-compact $M_{a}$, however, never reach any vacua and have infinite tension. Therefore they have to be discarded. Thus, the total moduli space $\mathcal{M}_{\text {wall }}$ of walls consists of the union of $M_{a}$ 's which is compact, that is,

$$
\mathcal{M}_{\text {wall }}=\bigcup_{K_{a}: \text { bounded }} M_{a}
$$

\subsection{Attraction and repulsion between BPS walls}

Let us move on to study the dynamics of BPS walls. Using the method in Ref. [54, repulsive force between two BPS walls was found [21] in the massive hyper-Kähler NLSM on the ALE target space with multi-center arranged on a single line ${ }^{8}$. As a preliminary to the later analysis, we first show that the similar repulsive force can occur in double walls interpolating three vacua $\alpha, \beta, \gamma$ arranged on a straight line in the $\mu_{A}$ space. Examples in the present paper are the two double wall configurations interpolating $\alpha_{1}, \alpha_{3}, \alpha_{5}$, and $\alpha_{2}, \alpha_{4}, \alpha_{5}$ in Fig. 4 in section 3.4. Interaction between two walls $\alpha \rightarrow \beta$ and $\beta \rightarrow \gamma$ turn out to be repulsive as follows. Each individual wall of the double wall is a BPS state preserving the same $1 / 2$ supersymmetry. Once placed side by side, however, the configuration $\alpha \rightarrow \beta \rightarrow \gamma$ is a non-BPS state. This is because the sign of one of $\mu_{A}$ 's must

\footnotetext{
${ }^{8}$ See also Introduction in Ref. [19] and 22]. A similar phenomenon has been also studied in a theory with four SUSY [55.
} 
be flipped at somewhere in such a configuration but it is prohibited by the BPS equations as was explained below Eq. (3.36). Unlike double walls which consist of a BPS wall and an anti-BPS wall, total energy density of such double walls is bounded below by a sum of the tension of each individual BPS wall. Therefore the total energy density must increase if we bring one of the BPS walls close to the other wall from the spatial infinity. We thus have shown that there exists repulsive force between two walls connecting vacua arranged on a straight line in the $\mu_{A}$ space.

The same discussion can be applied to other pairs of BPS walls, for instance, a set of wall $\alpha_{4} \rightarrow \alpha_{3}$ and $\alpha_{3} \rightarrow \alpha_{1}$ in the case I in subsection 3.4.1. Namely, there exists a repulsive force between these two walls. This repulsion is explained naturally if we deform the wall $\alpha_{4} \rightarrow \alpha_{3}$ to the double wall configurations made of the walls $\alpha_{4} \rightarrow \alpha_{5}$ and $\alpha_{5} \rightarrow \alpha_{3}$, because the two walls $\alpha_{5} \rightarrow \alpha_{3}$ and $\alpha_{3} \rightarrow \alpha_{1}$ are the case explained in the last paragraph. Indeed, there are no walls directly going from $\alpha_{4}$ to $\alpha_{1}$. In the same way we can find that there exists a repulsive force between walls $\alpha_{2} \rightarrow \alpha_{4}$ and $\alpha_{4} \rightarrow \alpha_{3}$. There turns out to be also attractive forces between certain sets of BPS walls as we will explain below.

In the generic massive hypertoric sigma model, there exist many sets of vacua arranged on a straight line in the $\mu_{A}$ space as in Fig. 2. Thus repulsion between BPS walls is not special, but one of commonplace features.

Let us now study in detail the dynamics of the BPS walls in Case I. Firstly, consider walls connecting $\alpha_{4}$ and $\alpha_{3}$. From the flow diagram, we can see that the wall moduli is complex two dimensional and that it can be identified with $W \mathbb{C} P^{2}{ }_{1,1, n}$. The walls can also be considered from the viewpoint of $T^{*} F_{n}$, since both the vacua $\alpha_{3,4}$ are on $F_{n}$. The Morse indices at the vacua of the flow inside $F_{n}$ are both two, as can be seen in (2.22) or (3.51). The wall moduli inside $F_{n}$ is of real dimension two, namely $\operatorname{dim}\left[S\left(\alpha_{3}\right) \cap U\left(\alpha_{4}\right)\right]=2$ where $S$ and $U$ denote stable and unstable manifolds, respectively, defined in Appendix A. When transversality holds this must coincides with $\operatorname{dim} S\left(\alpha_{3}\right)+\operatorname{dim} U\left(\alpha_{4}\right)-\operatorname{dim} F_{n}$. However the latter is now $\operatorname{dim} S\left(\alpha_{3}\right)+\operatorname{dim} U\left(\alpha_{4}\right)-\operatorname{dim} F_{n}=2+2-4=0 \neq 2=\operatorname{dim}\left[S\left(\alpha_{3}\right) \cap U\left(\alpha_{4}\right)\right]$. Therefore the flow violates transversality. According to the general discussion in section 2. there should be walls along the cotangent direction. We can now identify these walls with component along the cotangent direction as the walls inside $W \mathbb{C} P^{2}{ }_{1,1, n}$, thanks to the embedding to the hypertoric.

We can also see that there is another vacuum $\alpha_{5}$ outside the base $F_{n}$. It may be allowed to say that this extra vacuum $\alpha_{5}$ pulled the wall along the cotangent. Note that, although $\alpha_{5}$ is situated at the coordinate infinity along the cotangent direction, the geodesic distance from $F_{n}$ to $\alpha_{5}$ is finite. This addition of point at 'infinity' is very natural from the hypertoric point of view. It is also inevitable from the viewpoint of the cotangent bundle of generic toric manifolds. This is because, as mentioned in section 2.2 the hyper-Kähler metric on $T^{*} M$ is unique once one fix the metric on $M$ and placed the condition (*). Thus, one cannot help but add $\alpha_{5}$ at infinity.

Next let us study the wall interpolating $\alpha_{2}$ and $\alpha_{1}$. In a certain limit it becomes a compressed single wall along $\mu_{1}=0$ in Fig. 4. The moduli space with generic moduli 

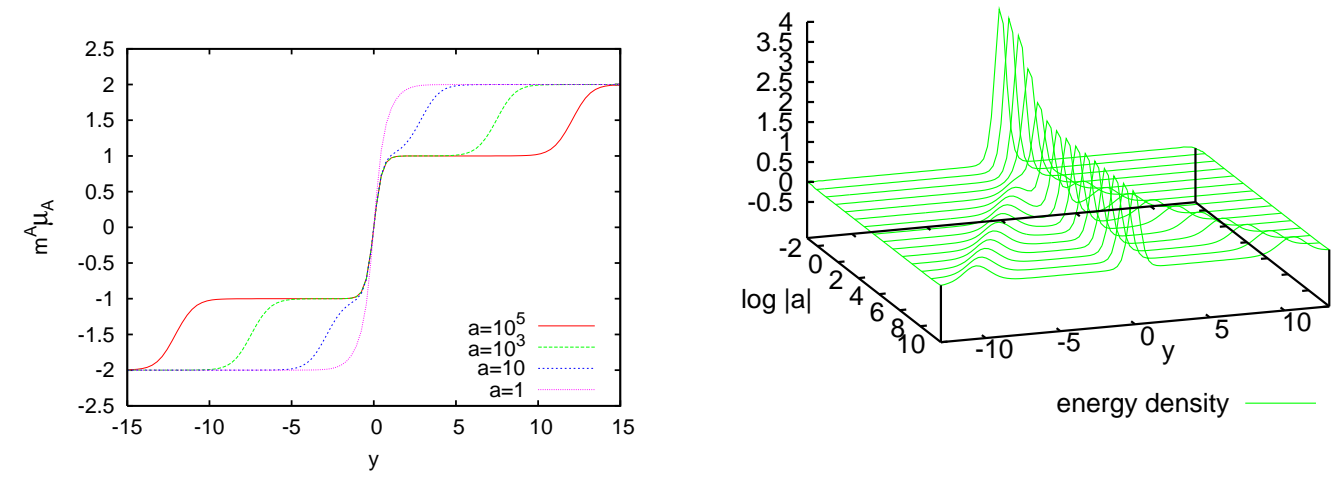

Figure 6: Profiles of walls $\alpha_{2} \rightarrow \alpha_{4} \rightarrow \alpha_{3} \rightarrow \alpha_{1}$. We plotted accumulated energy density $\int_{0}^{y} \mathcal{E} d y$ in the left, and energy density $\mathcal{E}$ in the right.
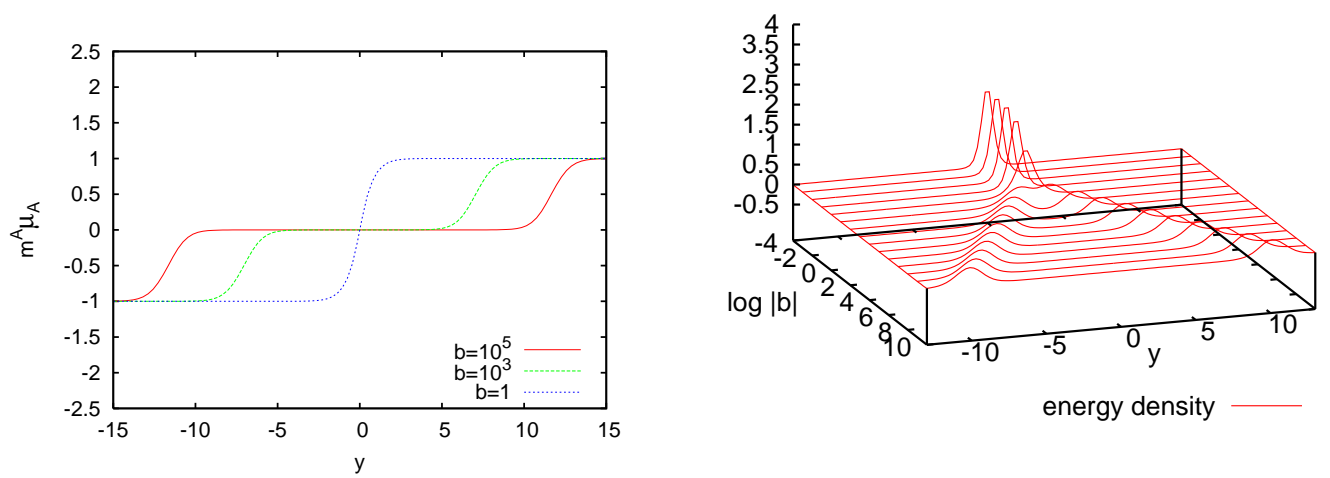

Figure 7: Profiles of walls $\alpha_{4} \rightarrow \alpha_{5} \rightarrow \alpha_{3}$. We plotted accumulated energy density $\int_{0}^{y} \mathcal{E} d y$ in the left, and energy density $\mathcal{E}$ in the right. 
parameters is contained in $F_{n}$ and is of complex two dimensions. Contrary to this, there exist three BPS walls interpolating $\alpha_{2} \rightarrow \alpha_{4}, \alpha_{4} \rightarrow \alpha_{3}$ and $\alpha_{3} \rightarrow \alpha_{1}$, and each wall carries a $U(1)$ phase. There exists one complex moduli parameter if we consider each of them independently. Therefore one might expect that there exist three complex moduli parameters for the configuration interpolating $\alpha_{2}$ and $\alpha_{1}$, whose number is not in agreement with the dimension of $F_{n}$. This apparent discrepancy can be resolved if we interpret that the wall $\alpha_{2} \rightarrow \alpha_{1}$ is in a sense a merger of three walls as follows. Indeed, in a certain range of moduli, there are clearly three spatial regions where energy is concentrated as depicted in Fig. 6. One can see there that the position of the inner wall is determined by the outer walls for the system to attain minimum energy. This can be understood as follows: First recall that there are repulsive force acting between a pair of walls $\alpha_{2} \rightarrow \alpha_{4}$ and $\alpha_{4} \rightarrow \alpha_{3}$ and between another pair $\alpha_{4} \rightarrow \alpha_{3}$ and $\alpha_{3} \rightarrow \alpha_{1}$, as discussed in the second paragraph in this subsection. Nevertheless there are BPS walls once we put in three walls. From these two facts we deduce that there must be attractive force between walls $\alpha_{2} \rightarrow \alpha_{4}$ and $\alpha_{3} \rightarrow \alpha_{1}$ in order to balance the repulsive interaction.

Finally consider separating the outer two walls to two spatial infinities in the three wall system above. Now, the remaining wall can be thought of as interpolating from $\alpha_{4}$ to $\alpha_{3}$. Although this wall is apparently a single wall system as exemplified in Fig. 17, there are additional moduli for the cotangent direction and we can make it split into a double wall configuration consisting of walls $\alpha_{4} \rightarrow \alpha_{5}$ and $\alpha_{5} \rightarrow \alpha_{3}$. The inner walls cannot be separated in the presence of outer walls $\alpha_{2} \rightarrow \alpha_{4}$ and $\alpha_{3} \rightarrow \alpha_{1}$. This fact suggests that the repulsive force from the outer two walls compresses the inner two walls to the single wall $\alpha_{4} \rightarrow \alpha_{3}$. The dividing process of a compressed single wall $\alpha_{2} \rightarrow \alpha_{1}$ to the three walls, and further dividing of the middle wall $\alpha_{4} \rightarrow \alpha_{3}$ to the two walls after taking off the outer two walls are shown in Fig. 8 .

In this system, the total wall moduli is the union of $M_{1}=F_{n}$ and $M_{2}=W \mathbb{C} P_{1,1, n}^{2}$. Their intersection is $\mathbb{C} P^{1}$, which is precisely the moduli space of walls $\alpha_{4} \rightarrow \alpha_{3}$, with the two vacua $\alpha_{3,4}$ added. This wall has the position $\operatorname{Re} \tau$ and the phase $\operatorname{Im} \tau$ associated with it as the only moduli. Let us look in more detail how $M_{1}$ and $M_{2}$ join at $\mathbb{C} P^{1}$. We take $\tau$ and $a$ as the coordinates of $M_{1}$, and $\tau$ and $b$ as those for $M_{2}$. As we have seen in (3.55) and (3.63), $\log |a|$ and $\log |b|$ control the relative position of constituent walls. Then, the intersection is the curve $1 / a=0$ in $M_{1}$ or $b=0$ in $M_{2}$, which is schematically illustrated in Fig. 9]

One can imagine a non-BPS wall configuration interpolating the all vacua successively as $\alpha_{2} \rightarrow \alpha_{4} \rightarrow \alpha_{5} \rightarrow \alpha_{3} \rightarrow \alpha_{1}$, as depicted in Fig. 10. Only when $1 / a=0$ i.e. two outer walls are infinitely far away, BPS walls with $b \neq 0$ can appear. Conversely, BPS walls with $|a|<\infty$ can arise only when $b=0$ i.e. two inner walls are completely compressed to a single wall. In this sense, one can consider $b \cdot(1 / a)=0$ as the defining equation of the whole BPS moduli space.

Although we used an explicit example containing $T^{*} F_{n}$ in order to illustrate the prop- 


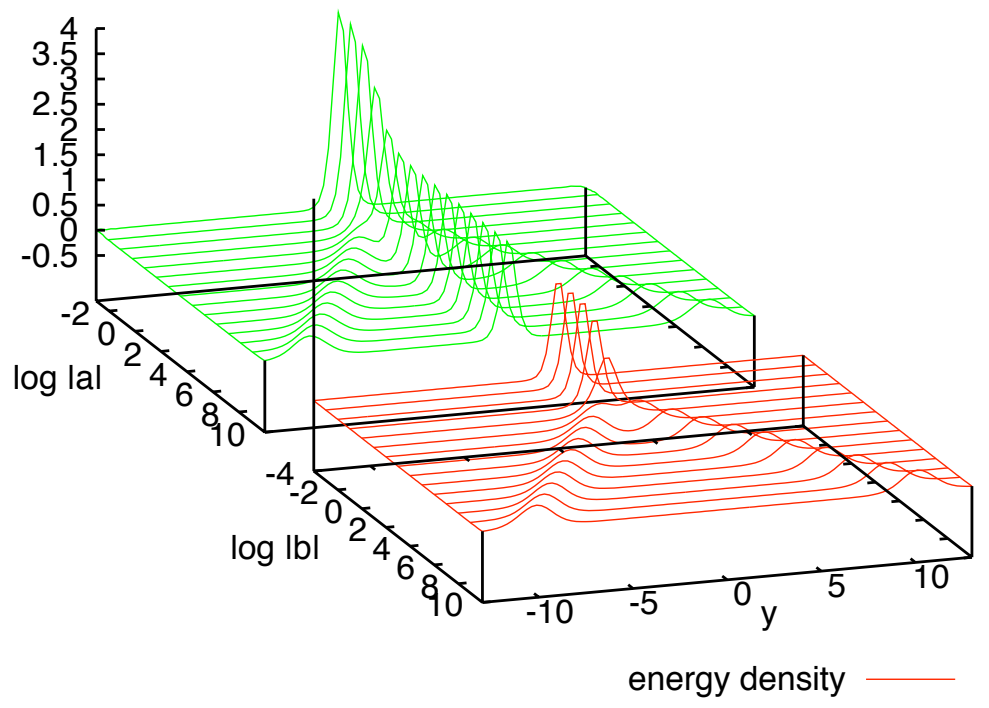

Figure 8: Walls breaking into constituents

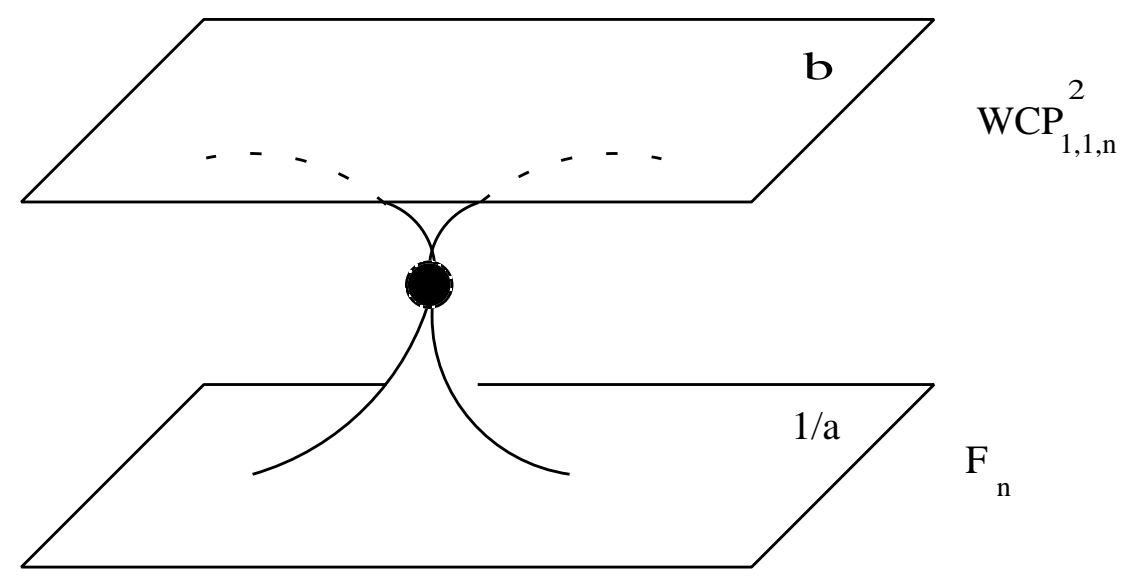

Figure 9: Schematic view of the intersection of $F_{n}$ and $W \mathbb{C} P_{1,1, n}^{2}$. The coordinate representing the center of mass is suppressed as $\mathcal{M}_{\text {wall }} / \mathbb{C}^{*}$. 


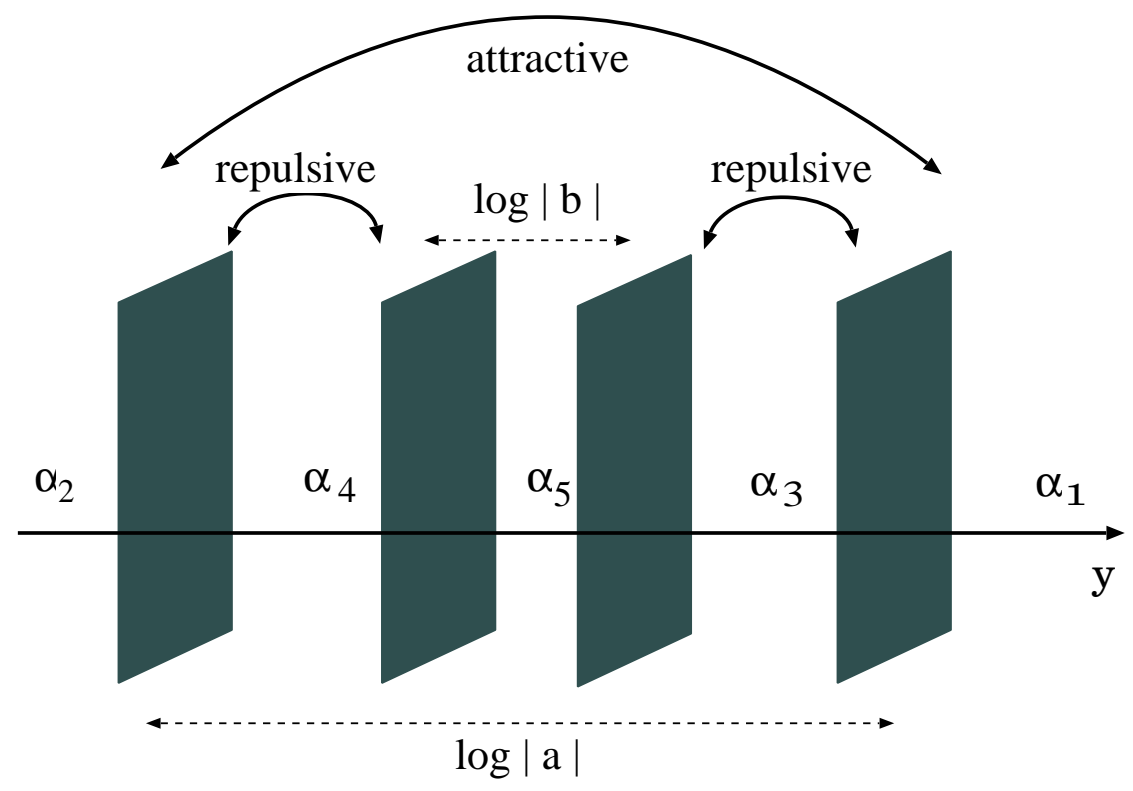

Figure 10: Schematic structure of walls interpolating from $\alpha_{2}$ to $\alpha_{1}$.

erties of walls, it is easy to see that these phenomena happen generically for any massive hyper-Kähler sigma model with hypertoric target spaces. Extra vacua at infinity along cotangent direction appear whenever the hyperplanes bounding the toric diagram of the base form an obtuse angle and intersect outside of the base. Simultaneously, there appear walls which flow outside the cotangent direction. It is extremely rare to have no obtuse angles. Indeed, any toric diagram with only acute or right angles necessarily corresponds to some direct product of weighted projective spaces. Thus, other than those cases, there are various violation of transversality and mutual attraction/repulsion between BPS walls.

\subsection{Transmutation of walls}

In the previous section we have focused on the case I of the gauged linear sigma model containing $F_{n}$, and have found interesting attraction and repulsion force between BPS walls. Although the cases II and III do not violate in themselves the transversality of the flow, yet there are fascinating dynamics between the BPS walls when they pass through each other.

Recall the case of the walls in the Grassmannians [29. There, certain pair of walls cannot cross each other, and when the moduli parameter for the relative distance is formally taken to be negative infinity the walls are made into a single compressed wall. Another pair of walls can pass through each other, and we called them penetrable wall system. The wall system before and after the crossing was equivalent, hence the walls retained their identities, like their tension. 

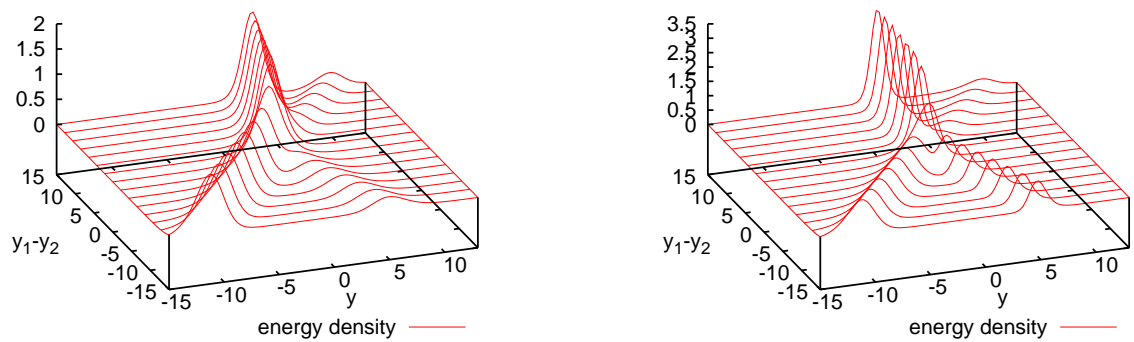

Figure 11: Transmutation of walls when they pass through. $m^{A}=(1,0,0,-1)$ in the left and $m^{A}=(2,0,0,-1)$ in the right.

The case studied in the last subsection is in a sense analogous to the compressed wall in Grassmannians. Now let us study the crossing of walls in $F_{n}$. It may not be surprising to the reader, who have read all the findings discussed in the previous sections, that we find richer dynamics in this case also.

Let us concentrate on the case II in subsection 3.4.2 and consider walls interpolating from vacua $\alpha_{2}$ to $\alpha_{3}$. We have worked out the wall tensions and wall positions for the case $M_{1}=F_{1}$ and the hypermultiplet masses $m^{A}=(q m, 0,0,-m)$ in section 3.4.2. The wall positions can be parametrized by $y_{i} \equiv \operatorname{Re} \tau_{i} / m$ using the moduli matrix parametrization in Eq. (3.68). We present the result of wall positions in Fig. 111 by taking $m=1$ and $q=1$ or $q=2$ as illustrative examples.

In the limit $y_{1}-y_{2} \rightarrow \infty$, the double wall system breaks into two walls $W_{1}: \alpha_{2} \rightarrow \alpha_{4}$ and $W_{2}: \alpha_{4} \rightarrow \alpha_{3}$. In the limit $y_{1}-y_{2} \rightarrow-\infty$, on the other hand, the system breaks into $W_{1}^{\prime}: \alpha_{2} \rightarrow \alpha_{1}$ and $W_{2}^{\prime}: \alpha_{1} \rightarrow \alpha_{3}$. If $y_{1} \sim y_{2}$, the two walls are merged into one wall $W$. The process can clearly be seen in Fig. 11. What distinguishes this case and the previously studied examples of walls in Grassmannians or projective spaces is that now the soliton before and after the encounter, $W_{1}$ and $W_{2}^{\prime}$, is not equivalent. $W_{2}$ and $W_{1}^{\prime}$ also cannot be transformed to each other. Hence, the BPS walls transmuted through the encounter. In the case $m^{A}=(1,0,0,-1)$, the tension of the walls $W_{1}$ and $W_{1}^{\prime}$ accidentally equals to each other as can be seen from Table 2. In the case $m^{A}=(2,0,0,-1)$ however, the tension of walls $W_{1,2}$ and $W_{1,2}^{\prime}$ differs from each other completely with the sum of the tension unchanged. Thus there is no doubt that the soliton has changed its identity.

\section{Conclusion and discussion}

In this paper, we have analyzed the BPS flow equation of massive NLSMs with eight supercharges for various hyper-Kähler target spaces. We first have treated the most generic hyper-Kähler manifold, and then have studied the case of $T^{*} M$ with Kähler manifolds $M$ 
and finally hypertoric manifolds, with increasing amount of detail in this order.

It is still difficult to survey the whole structure of general hyper-Kähler sigma models. However, we have been able to see at least the connection of the index theorem and the problem of transversality of the BPS flow equation for the models with the $T^{*} M$ target space. In addition, once we restricted attention to hypertoric sigma models, we have found that the total moduli space of BPS walls, containing all possible topological sectors, is precisely the core of the hypertoric manifold, which is the union of the special Lagrangian toric compact submanifolds defined by the charge matrix for the hypertoric manifold.

We also have found that the essential reason for the non-transversality of the flow is that the hyperplane arrangement for $T^{*} F_{n}$ contains a trapezoidal cell $K_{1}$ with an obtuse angle, representing $M_{1}=F_{n}$ in Fig. 4. Due to this, another vacuum $\alpha_{5}$ appears outside of the base $F_{n}$ and it is on another compact special Lagrangian submanifold $M_{2}=W \mathbb{C} P_{1,1, n}^{2}$ neighboring $F_{n}$. In such a case, sets of vacua, $\left(\alpha_{2}, \alpha_{4}, \alpha_{5}\right)$ and $\left(\alpha_{1}, \alpha_{3}, \alpha_{5}\right)$, are arranged along a straight line in the $\mu_{A}$ space, and this is the cause of the mutual repulsion among BPS walls.

Directions of BPS flows are classified into three cases as in Fig. 5, depending on the arrangement of the hypermultiplet masses. The violation of the transversality can be seen in the case I but not in the cases of II and III. We have found in the case I that, in the triple wall sector, the position of the center wall is locked in the center between the outer two walls as in Fig. 6. We then have deduced the existence of the mutual attraction and repulsion between the walls as summarized in Fig. 10. It would be extremely interesting to elucidate the dynamical mechanism leading to those forces. On the other hand, in the other two cases, the same feature of the $T^{*} F_{n}$ model, namely, the existence of multiple compact special Lagrangian submanifolds, causes completely different phenomenon, the transmutation of walls: when two walls path through, tension of one wall is transfered to the other with the sum of their tension unchanged, as illustrated in Fig. 11] So we have obtained these interesting dynamics of walls for the $T^{*} F_{n}$ model from the same geometrical characteristic.

The total moduli space is the union of $F_{n}$ and $W \mathbb{C} P^{2}{ }_{1,1, n}$ which join together sharing the submanifold $\mathbb{C} P^{1}$ as schematically illustrated in Fig. 9. We would like to emphasize that we never see the global structure of the moduli space like this if we restrict ourselves only to each topological sector.

We expect that similar analysis can be extended to more general and complicated hypertoric sigma models. If the model contains $n$-polygons $(n \geq 4)$ in the hyperplane arrangement, the non-transversality and transmutation may occur to walls connecting vertices of one of the polygons.

One of interesting future directions of research will be the study on the dynamics of the wall moduli. As in the work by Atiyah and Hitchin [56] on the scattering of BPS monopoles, we should be able to study the scattering of BPS walls with each other because the Kähler potential of the moduli space can be obtained easily as done in Ref. [29] in 
the case of Grassmannian. This will be particularly interesting when the transmutation of walls occur. Extending the discussion to the quantum case will be also worthwhile. Since the worldvolume theory has only four supercharges, there will be non-perturbative superpotential generated by BPS solitons of the effective theory. These solitons are precisely the vortices of the original theory, and their moduli space has been already analyzed in 30. Hence, the calculation à la Affleck, Dine and Seiberg [57] will in principle be possible. We can expect richer non-perturbative dynamics of the BPS walls.

BPS walls in the case of Grassmannian NLSM can be realized using kinky D $p$-brane configuration in the background $\mathrm{D}(p+4)$-branes on the ALE space 32]. That D-brane picture was extremely useful to understand various dynamics of walls. Therefore D-brane construction for the models discussed in the present paper is desirable. In particular it would be interesting to understand complicated dynamics of walls like the wall locking or the transmutation found in the present paper.

Finally, we would like to recall that much has been gained from taking the Killing potential of the holomorphic isometry as the Morse function. Combined with the restriction which comes from the eight supercharges, we should be able to extract more geometrical information from the BPS flow structure on hyper-Kähler target spaces. We are planning to investigate these problems further.

\section{Acknowledgement}

This work is supported in part by Grant-in-Aid for Scientific Research from the Ministry of Education, Culture, Sports, Science and Technology, Japan No.13640269 (NS) and 16028203 for the priority area "origin of mass" (NS). The works of K.Ohashi and M.N. are supported by Japan Society for the Promotion of Science (JSPS) under the Post-doctoral Research Program while the work of Y.T. is by the JSPS DC1 program. The works of Y.I. and M.E. are supported by a 21st Century COE Program at Tokyo Tech "NanometerScale Quantum Physics" by the Ministry of Education, Culture, Sports, Science and Technology. M. E. also gratefully acknowledges support from the Iwanami Fujukai Foundation. K. Ohta is supported in part by Special Postdoctoral Researchers Program at RIKEN.

\section{A Transversality and the Morse-Smale condition}

In this appendix we collect facts about the Morse-Smale condition and transversality. 


\section{A.1 Stable and unstable manifolds}

Let $M$ be a compact manifold with dimension $n, f$ be a Morse function and $\alpha, \beta, \cdots$ be critical points of $f$. Let $g$ be a metric on $M$. Define the Morse flow $\varphi_{y}(p)$ with parameter $y$ with respect to $f$ as the solution of the differential equation

$$
\frac{d}{d y} \varphi_{y}(p)=g^{i j} \partial_{j} f\left(\varphi_{y}(p)\right)
$$

with the initial condition $\varphi_{0}(p)=p$. Then, stable and unstable manifolds of $\alpha$ are defined as follows:

$$
\begin{aligned}
S(\alpha) & =\left\{p \in M \mid \lim _{y \rightarrow+\infty} \varphi_{y}(p)=\alpha\right\}, \\
U(\alpha) & =\left\{p \in M \mid \lim _{y \rightarrow-\infty} \varphi_{y}(p)=\alpha\right\},
\end{aligned}
$$

respectively. These $S(\alpha)$ and $U(\alpha)$ are submanifolds of $M$ and homeomorphic to $\mathbb{R}^{n-n_{\alpha}}$ and $\mathbb{R}^{n_{\alpha}}$ respectively, and $n_{\alpha}$ is called the Morse index of $\alpha$. If we change the sign of the Morse function from $f$ to $-f$, the definition of stable and unstable manifold is exchanged. The Morse function $f$ on $M$ defines the following two kinds of decompositions. First one is

$$
M=\bigcup_{\alpha} S(\alpha)=\bigcup_{\alpha} U(\alpha)
$$

where $\alpha$ is summed over all critical points of $f$. So $M$ is decomposed into a sum of disjoint cells $S(\alpha)$ or $U(\alpha)$. Second decomposition is

$$
M=\bigcup_{\alpha, \beta} F(\alpha, \beta), \quad F(\alpha, \beta) \equiv S(\alpha) \cap U(\beta),
$$

where the sum is taken over all critical points $\alpha, \beta$. Namely, $M$ is the sum of walls which go from $\beta$ to $\alpha$.

\section{A.2 Morse-Smale transversality condition}

$S(\alpha)$ intersect transversely with $U(\beta)$ if and only if the following condition is satisfied for all critical points $\alpha, \beta$ of $f$ :

$$
T_{p} S(\alpha)+T_{p} U(\beta)=T_{p} M, \text { for all } p \in S(\alpha) \cap U(\beta) .
$$

This condition is called the Morse-Smale condition and then $f$ is called the Morse-Smale function on $M$. In this case, since $S(\alpha) \cap U(\beta)$ is submanifold of $M$,

$$
\operatorname{dim}[S(\alpha) \cap U(\beta)]=\operatorname{dim} S(\alpha)+\operatorname{dim} U(\beta)-\operatorname{dim} M,
$$

so that we find

$$
\operatorname{dim} F(\alpha, \beta)=\left(n-n_{\alpha}\right)+n_{\beta}-n=n_{\beta}-n_{\alpha},
$$

where $n_{\alpha, \beta}$ are indices of $\alpha, \beta$. 


\begin{tabular}{c|cccccccc} 
ours & $\partial_{y}$ & $\Sigma$ & $\mathcal{D}_{1}$ & $\mathcal{D}_{2}$ & $g^{2}$ & $c$ & $\sigma$ & $\eta$ \\
Ref.[36] & $\partial_{3}$ & $\zeta^{T}$ & $\Delta^{\dagger}$ & $\Delta$ & $e^{2}$ & $v^{2}$ & $\alpha$ & $\beta$
\end{tabular}

Table 3: Comparison of notations with Ref. 36

\section{B On the absence of the vanishing theorem}

In the main part of the article, we saw that the BPS flow inside $F_{n}$ violates transversality for certain range of mass parameters. Additionally, we explicitly constructed a gauged linear sigma model with eight supersymmetry which contains $F_{n}$ as a special Lagrangian submanifold. Using the notation of section [3.4 the BPS flow inside $F_{n}$ starting from $\alpha_{4}$ to $\alpha_{3}$ connects the vacua with the same Morse index. As we showed in section [2, this violation of transversality is related to the non-vanishing of the zero modes of the operator $\mathcal{D}_{1}$, which is the adjoint of the operator $\mathcal{D}_{2}$ governing the deformation of the flow inside the base.

Those who also read the papers [33] and 36, however, may wonder why such a situation occurs, because it was shown therein that there were no such zero modes for gauge groups $U(1)$ and $S U\left(N_{c}\right)$, respectively. We would like to clarify why the naïve extension of the argument in those papers fails in our case. As the exposition closely follows that of 36, please place the paper side-by-side and compare with it. The notation difference is tabulated in Table 3. We emphasize that the various phenomena such as violation of transversality, can be regarded as one and the same phenomena as the absence of the vanishing theorem.

Let us take a massive Abelian gauged linear sigma model with several $U(1)$ factor groups. Since the gauge coupling $g_{I}$ can be absorbed into the redefinition of the hypermultiplet charges $q_{I}^{A}$, we will choose to use $2 g_{I}^{2}=1$. The equations for the fluctuations around the BPS wall solution can be obtained by perturbing the BPS equations following the argument in [36]. Denoting the fluctuations of $\Sigma^{I}+i W_{y}^{I}, h_{A}$ and $\tilde{h}^{A}$ as $\sigma^{I}, \eta_{A}$ and $\tilde{\eta}^{A}$, respectively, we find after using the Gauss law and fixing the gauge

$$
\begin{aligned}
-\partial_{y} \sigma^{I}-\sum_{A} q_{I}^{A}\left(h^{A \dagger} \eta_{A}-\tilde{h}_{A}^{\dagger} \tilde{\eta}^{A}\right)=0 & (\text { for each } I), \\
\left(-\partial_{y}-\sum_{I} q_{I}^{A} \Sigma^{I}+m^{A}\right) \eta_{A}-\sum_{I} q_{I}^{A} h_{A} \sigma^{I}=0 & (\text { for each } A), \\
\left(-\partial_{y}+\sum_{I} q_{I}^{A} \Sigma^{I}-m^{A}\right) \tilde{\eta}^{A}+\sum_{I} q_{I}^{A} \tilde{h}^{A} \sigma^{I}=0 & (\text { for each } A) .
\end{aligned}
$$

As shown in section 2, we should consider a special Lagrangian submanifold to obtain a nontrivial index. Here let us take a flow on the special Lagrangian submanifold $M$ defined by $\tilde{h}^{A}=0$. By disregarding the fluctuation $\tilde{\eta}^{A}$ and setting $\tilde{h}^{A}=0$ in Eqs.(B.1)-(B.3), we 
obtain the operator $\mathcal{D}_{2}$ in (2.12) as

$$
\mathcal{D}_{2}=\left(\begin{array}{cc}
-\partial_{y} \mathbb{1} & -q_{I}^{A} h_{A}^{\dagger} \\
-q_{I}^{A} h_{A} & \operatorname{diag}\left(-\partial_{y}-\sum_{I} q_{I}^{A} \Sigma^{I}+m^{A}\right)
\end{array}\right),
$$

acting on a vector $\left(\sigma^{I}, \eta_{A}\right)^{T}$. Here $\mathbb{1}$ is the unit matrix acting on the color indices, and $q_{I}^{A} h_{A}$ should be thought of as a $N \times N_{F}$ matrix. The operator $\mathcal{D}_{1}$ is obtained as the adjoint of the operator $\mathcal{D}_{2}$ as

$$
\mathcal{D}_{1}=\left(\begin{array}{cc}
\partial_{y} \mathbb{1} & -q_{I}^{A} h_{A}^{\dagger} \\
-q_{I}^{A} h_{A} & \operatorname{diag}\left(\partial_{y}-\sum_{I} q_{I}^{A} \Sigma^{I}+m^{A}\right)
\end{array}\right)
$$

Let us try to show that there is no zero modes of the operator $\mathcal{D}_{1}$, imitating the argument in [36]. Then we have

$$
\begin{aligned}
& \left|\mathcal{D}_{1}\left(\begin{array}{c}
\sigma^{I} \\
\eta_{A}
\end{array}\right)\right|^{2}=\left|\partial_{y} \sigma^{I}\right|^{2}+\left|\sum_{A} q_{I}^{A} h_{A}^{\dagger} \eta_{A}\right|^{2} \\
& +\left|\partial_{y} \eta_{A}-\sum_{I} \eta_{A} q_{I}^{A} \Sigma^{I}+\eta_{A} m^{A}\right|^{2}+\left|\sum_{I} q_{I}^{A} h_{A} \sigma_{I}\right|^{2}+\text { X-terms }
\end{aligned}
$$

and the X-terms vanish when one uses the BPS equation, just as in 36. Hence, the following four conditions are necessary and sufficient for $\left(\sigma^{I}, \eta_{A}\right)^{T}$ to be a zero mode:

$$
\begin{aligned}
\partial_{y} \sigma^{I} & =0 & & (\text { for each } I), \\
\sum_{I} q_{I}^{A} h_{A} \sigma_{I} & =0 & & (\text { for each } A), \\
\sum_{A} q_{I}^{A} h_{A}^{\dagger} \eta_{A} & =0 & & (\text { for each } I), \\
\partial_{y} \eta_{A}-\sum_{I} \eta_{A} q_{I}^{A} \Sigma^{I}+\eta_{A} m^{A} & =0 & & (\text { for each } A) .
\end{aligned}
$$

Eqs. (B.7) and (B.8) immediately dictate the fluctuation of vector multiplet scalar to vanish $\sigma^{I}=0$. Let us now concentrate on Eqs. (B.9) and (B.10) to examine if the fluctuation $\eta_{A}$ of the hypermultiplet scalar $h_{A}$ has zero mode or not. The 'moduli matrix' $q_{I}^{A} h_{A}$ is just a row vector, and the condition (B.9) imposes only $N$ conditions on the $N_{f}$ variables ${ }^{9}$. Hence Eq. (B.9) is not sufficient to conclude that $\eta$ vanishes. The equation (B.9) is just the condition (3.19) which signifies that $\eta$ should be cotangent to the base manifold. Thus, we found again that the adjoint of the operator governing the deformation of the BPS flow inside the base describes the BPS flow along the cotangent direction.

\footnotetext{
${ }^{9}$ A similar analysis in Ref. 33 contains an incorrect result in its Eq.(15) with respect to this point. Therefore the proof of the vanishing theorem in that case also requires the argument that we give in this Appendix.
} 
We can do better in sorting out the zero modes of $\mathcal{D}_{1}$. It is important to realize that the remaining zero mode equation (B.10) for each $A$ is identical to the equation for the fluctuation $\tilde{\eta}^{A}$ of the cotangent direction $\tilde{h}^{A}$ in Eq.(B.3), provided we can neglect the background $\tilde{h}^{A}=0$ or the fluctuation of vector multiplet scalar $\sigma^{I}=0$. This again shows that the vanishing theorem (no zero mode of $\mathcal{D}_{1}$ ) is the same condition as the absence of the zero mode in the cotangent direction, even for finite gauge coupling rather than the NLSM (strong gauge coupling) limit. Eq. (B.10) combined with the BPS equation (3.31) implies that the quantities $h_{A} \tilde{\eta}^{A}$ (no summation on $A$ ) are constant along the flow. Since $h_{A} \tilde{\eta}^{A}$ at $y \rightarrow \pm \infty$ are constrained to vanish, $h_{A} \tilde{\eta}^{A}$ (no summation on $A$ ) should always be zero, similarly to $\nu_{A}$ as noted already in (3.36). Thus, when $h_{A}$ is nonzero for a particular flavor $A$, there is no zero mode $\tilde{\eta}^{A}$ along the corresponding $\tilde{h}^{A}$ direction. Let us note that the condition $h_{A}$ vanishes is equivalent to the condition $\mu_{A}=h_{A} h_{A}^{\dagger}$ vanishes, and recall that the hyperplanes $\mu_{A}=0$ define the boundary of the cell corresponding to the special Lagrangian submanifold we are considering. Therefore, we can conclude that the vanishing theorem can be established when the flow is properly inside the cell, and the zero mode along the cotangent direction is possible only for the flow along the boundary of the cell.

When there is indeed a nontrivial zero mode along $\tilde{h}^{A}$, the same condition $h_{A} \tilde{\eta}^{A}=0$ (no summation on $A$ ) now implies $h_{A}=0$ for that particular $A$. This means that the BPS flow in this particular topological sector lost one moduli inside the base. Additionally, the flow with nonzero $\tilde{h}^{A}$ can be naturally considered as the flow inside the other special Lagrangian submanifold $M^{\prime}$ which shares the hyperplane $\mu_{A}=0$. Thus, for the flow inside the hypertorics, the appearance of the zero mode along the cotangent direction in a topological sector can be better understood as the existence of another compact special Lagrangian submanifold $M^{\prime}$ sharing a boundary with $M$. Then, the wall in the particular topological sector is better interpreted as a result of taking the limit $\tilde{h}^{A} \rightarrow 0$ for walls inside $M^{\prime}$. Indeed, if we choose to consider the index theorem with respect to the special Lagrangian submanifold $M^{\prime}$ instead of $M$, we have a valid vanishing theorem and no violation of transversality. This point is very well illustrated in the discussion in section 3.6. There, the wall connecting $\alpha_{4}$ to $\alpha_{3}$ violated transversality and looked like a single wall from the viewpoint of $F_{n}$. The wall is, however, decomposable further into two walls $\alpha_{4} \rightarrow \alpha_{5}$ and $\alpha_{5} \rightarrow \alpha_{3}$ from the viewpoint of $W \mathbb{C} P_{1,1, n}^{2}$. This example strengthens our interpretation that the walls violating transversality should be thought of as a limit of the wall on another special Lagrangian submanifold.

We have shown in section 2.1 that Morse index for the entire hyper-Kähler manifold is always $2 n$ and does not give informations on the possible number of zero modes (wall directions). These NLSM's may be obtained by taking the strong gauge coupling limit from our gauged linear sigma model. We can obtain the corresponding result at finite gauge couplings by extending our analysis of zero modes as follows. Suppose we apply the same type of analysis of zero modes for index theorems by choosing the fluctuations $\sigma^{I}, \eta_{A}, \tilde{\eta}^{A}$ of the entire gauged linear sigma model. The operator $\mathcal{D}_{2}$ in (2.13) can be read off from Eqs. (B.7)-(B.10). The adjoint operator $\mathcal{D}_{1}$ can be easily worked out to give the same operator as $\mathcal{D}_{2}$ except that the sign of $\partial_{y}$ is just reversed. If we apply 
the same zero mode analysis as above for this case, we first find that vector multiplet scalar fluctuations should vanish. Then the remaining zero mode equations for $\mathcal{D}_{2}$ become $\partial_{y} \eta_{A}-\sum_{I} \eta_{A} q_{I}^{A} \Sigma^{I}+\eta_{A} m^{A}=0$ and $\partial_{y} \tilde{\eta}_{A}+\sum_{I} \tilde{\eta}_{A} q_{I}^{A} \Sigma^{I}-\tilde{\eta}_{A} m^{A}=0$. It is easy to see that these equations are identical to the corresponding zero mode equations for the operator $\mathcal{D}_{1}$, since the two equations are interchanged under $\partial_{y} \leftrightarrow-\partial_{y}$. This result implies that the index in Eq. (2.13) always vanishes if it is defined for the entire fluctuations of the gauged linear sigma model.

\section{References}

[1] E. B. Bogomol'nyi, Sov. J. Nucl. Phys. 24, 449 (1976) [Yad. Fiz. 24, 861 (1976)] ; M. K. Prasad and C. M. Sommerfield, Phys. Rev. Lett. 35, 760 (1975).

[2] E. Witten and D. I. Olive, Phys. Lett. B 78, 97 (1978).

[3] P. Horava and E. Witten, Nucl. Phys. B 460, 506 (1996) arXiv:hep-th/9510209.

[4] N. Arkani-Hamed, S. Dimopoulos and G. R. Dvali, Phys. Lett. B 429, 263 (1998) arXiv:hep-ph/9803315; I. Antoniadis, N. Arkani-Hamed, S. Dimopoulos and G. R. Dvali, Phys. Lett. B 436, 257 (1998) arXiv:hep-ph/9804398.

[5] L. Randall and R. Sundrum, Phys. Rev. Lett. 83, 3370 (1999) arXiv:hep-ph/9905221; Phys. Rev. Lett. 83, 4690 (1999) arXiv:hep-th/9906064.

[6] M. F. Atiyah, N. J. Hitchin, V. G. Drinfeld and Yu. I. Manin, Phys. Lett. A 65, 185 (1978).

[7] W. Nahm, Phys. Lett. B 90, 413 (1980).

[8] A. Hanany and D. Tong, JHEP 0307, 037 (2003) arXiv:hep-th/0306150; JHEP 0404, 066 (2004) arXiv:hep-th/0403158.

[9] M. Eto, M. Nitta and N. Sakai, Nucl. Phys. B 701, 247 (2004) arXiv:hep-th/0405161.

[10] R. Auzzi, S. Bolognesi, J. Evslin, K. Konishi and A. Yung, Nucl. Phys. B 673, 187 (2003) arXiv:hep-th/0307287.

[11] G. R. Dvali and M. A. Shifman, Nucl. Phys. B 504, 127 (1997) arXiv:hep-th/9611213; Phys. Lett. B 396, 64 (1997) [Erratum-ibid. B 407, 452 (1997)] arXiv:hep-th/9612128; A. Kovner, M. A. Shifman and A. Smilga, Phys. Rev. D 56, 7978 (1997) arXiv:hep-th/9706089; A. Smilga and A. Veselov, Phys. Rev. Lett. 79, 4529 (1997) arXiv:hep-th/9706217|; D. Bazeia, H. Boschi-Filho and F. A. Brito, JHEP 9904, 028 (1999) arXiv:hep-th/9811084; V. S. Kaplunovsky, J. Sonnenschein and S. Yankielowicz, Nucl. Phys. B 552, 209 (1999) arXiv:hep-th/9811195; G. R. Dvali, G. Gabadadze and Z. Kakushadze, Nucl. Phys. B 562, 158 (1999) 
arXiv:hep-th/9901032; M. Naganuma and M. Nitta, Prog. Theor. Phys. 105, 501 (2001) arXiv:hep-th/0007184; D. Binosi and T. ter Veldhuis, Phys. Rev. D 63, 085016 (2001) arXiv:hep-th/0011113; N. Maru, N. Sakai, Y. Sakamura and R. Sugisaka, Nucl. Phys. B 616, 47 (2001) arXiv:hep-th/0107204.

[12] M. A. Shifman, Phys. Rev. D 57, 1258 (1998) arXiv:hep-th/9708060; M. A. Shifman and M. B. Voloshin, Phys. Rev. D 57, 2590 (1998) arXiv:hep-th/9709137.

[13] B. S. Acharya and C. Vafa, arXiv:hep-th/0103011.

[14] A. Ritz, M. Shifman and A. Vainshtein, Phys. Rev. D 66, 065015 (2002) arXiv:hep-th/0205083; Phys. Rev. D 70, 095003 (2004) arXiv:hep-th/0405175; A. Ritz, JHEP 0310, 021 (2003) arXiv:hep-th/0308144.

[15] M. Cvetic, F. Quevedo and S. J. Rey, Phys. Rev. Lett. 67, 1836 (1991); M. Cvetic, S. Griffies and S. J. Rey, Nucl. Phys. B 381, 301 (1992) arXiv:hep-th/9201007.

[16] E. R. C. Abraham and P. K. Townsend, Phys. Lett. B 291, 85 (1992); Phys. Lett. B 295, 225 (1992).

[17] J. P. Gauntlett, D. Tong and P. K. Townsend, Phys. Rev. D 63, 085001 (2001) arXiv:hep-th/0007124.

[18] J. P. Gauntlett, R. Portugues, D. Tong and P. K. Townsend, Phys. Rev. D 63, 085002 (2001) arXiv:hep-th/0008221.

[19] J. P. Gauntlett, D. Tong and P. K. Townsend, Phys. Rev. D 64, 025010 (2001) arXiv:hep-th/0012178.

[20] D. Tong, Phys. Rev. D 66, 025013 (2002) arXiv:hep-th/0202012.

[21] D. Tong, JHEP 0304, 031 (2003) arXiv:hep-th/0303151.

[22] A. Opfermann, Cambridge University Ph.D Thesis, 1999.

[23] M. Arai, M. Naganuma, M. Nitta and N. Sakai, Nucl. Phys. B 652, 35 (2003) arXiv:hep-th/0211103; Garden of Quanta - In honor of Hiroshi Ezawa, Eds. by J. Arafune et al. (World Scientific Publishing Co. Pte. Ltd. Singapore, 2003) pp 299-325, arXiv:hep-th/0302028.

[24] M. Arai, E. Ivanov and J. Niederle, Nucl. Phys. B 680, 23 (2004) arXiv:hep-th/0312037.

[25] Y. Isozumi, K. Ohashi, and N. Sakai, JHEP 0311, 060 (2003) arXiv:hep-th/0310189; JHEP 0311, 061 (2003) arXiv:hep-th/0310130.

[26] K. Kakimoto and N. Sakai, Phys. Rev. D 68, 065005 (2003) arXiv:hep-th/0306077. 
[27] M. Arai, S. Fujita, M. Naganuma and N. Sakai, Phys. Lett. B 556, 192 (2003) arXiv:hep-th/0212175; to appear in the proceedings of International Seminar on Supersymmetries and Quantum Symmetries SQS 03, Dubna, Russia, 24-29 Jul 2003, arXiv:hep-th/0311210; to appear in the proceedings of SUSY 2003, "SUSY in the Desert" 11th Annual International Conference on Supersymmetry and the Unification of Fundamental Interactions, Tucson, Arizona, 5-10 Jun 2003. arXiv:hep-th/0402040; M. Eto, S. Fujita, M. Naganuma and N. Sakai, Phys. Rev. D 69, 025007 (2004) arXiv:hep-th/0306198.

[28] Y. Isozumi, M. Nitta, K. Ohashi and N. Sakai, Phys. Rev. Lett. 93, 161601 (2004) arXiv:hep-th/0404198.

[29] Y. Isozumi, M. Nitta, K. Ohashi and N. Sakai, Phys. Rev. D70, 125014 (2004), arXiv:hep-th/0405194.

[30] Y. Isozumi, M. Nitta, K. Ohashi and N. Sakai, to appear in Phys. Rev. D arXiv:hep-th/0405129.

[31] Y. Isozumi, M. Nitta, K. Ohashi and N. Sakai, to appear in the proceedings of 12th International Conference on Supersymmetry and Unification of Fundamental Interactions (SUSY 04), Tsukuba, Japan, 17-23 Jun 2004 arXiv:hep-th/0409110; to appear in the proceedings of "NathFest" at PASCOS conference, Northeastern University, Boston, Ma, August 2004 arXiv:hep-th/0410150.

[32] M. Eto, Y. Isozumi, M. Nitta, K. Ohashi, K. Ohta and N. Sakai, arXiv:hep-th/0412024.

[33] K. S. M. Lee, Phys. Rev. D 67, 045009 (2003) arXiv:hep-th/0211058.

[34] M. Eto, Y. Isozumi, M. Nitta, K. Ohashi and N. Sakai, arXiv:hep-th/0412048.

[35] M. Naganuma, M. Nitta and N. Sakai, Grav. Cosmol. 8, 129 (2002) arXiv:hep-th/0108133; R. Portugues and P. K. Townsend, JHEP 0204, 039 (2002) arXiv:hep-th/0203181.

[36] N. Sakai and D. Tong, to appear in JHEP, arXiv:hep-th/0501207.

[37] B. Zumino, Phys. Lett. B 87, 203 (1979); L. Alvarez-Gaumé and D. Z. Freedman, Commun. Math. Phys. 80, 443 (1981).

[38] L. Alvarez-Gaumé and D. Z. Freedman, Commun. Math. Phys. 91, 87 (1983).

[39] U. Lindström and M. Roček, Nucl. Phys. B 222, 285 (1983).

[40] N. J. Hitchin, A. Karlhede, U. Lindström and M. Roček, Commun. Math. Phys. 108, 535 (1987). 
[41] M. Arai, M. Nitta and N. Sakai, Prog. Theo. Phys. (in press) arXiv:hep-th/0307274; to appear in the Proceedings of the 3rd International Symposium on Quantum Theory and Symmetries (QTS3), September 10-14, 2003, arXiv:hep-th/0401084; to appear in the Proceedings of the International Conference on "Symmetry Methods in Physics (SYM-PHYS10)" held at Yerevan, Armenia, 13-19 Aug. 2003 arXiv:hep-th/0401102; to appear in the Proceedings of SUSY 2003 held at the University of Arizona, Tucson, AZ, June 5-10, 2003 arXiv:hep-th/0402065.

[42] G. Sierra and P. K. Townsend, Nucl. Phys. B 233, 289 (1984).

[43] J. Scherk and J.H. Schwarz, Phys. Lett. B 82, 60 (1979).

[44] D. Kaledin, arXiv:alg-geom/9710026; B. Feix, J. reine angew. Math. 532, 33 (2001).

[45] S. J. J. Gates and S. M. Kuzenko, Nucl. Phys. B 543, 122 (1999) arXiv:hep-th/9810137; Fortsch. Phys. 48, 115 (2000) arXiv:hep-th/9903013.

[46] C. Callias, Commun. Math. Phys. 62, 213 (1978).

[47] M. F. Atiyah, V. K. Patodi and I. M. Singer, Math. Proc. Cambridge Phil. Soc. 77, $43(1975)$;

[48] A. Białynicki-Birula, Bull. l'Acad. Polonaise Sci. 24, 667 (1976).

[49] R. Bielawski and A. Dancer, Comm. Anal. Geom. 8, 727 (2000); T. Hausel and B. Sturmfels, Doc. Math. 7, 495 (2002) arXiv:math.AG/0203096; M. Harada and N. J. Proudfoot, Pacific J. Math. 214, 263 (2004) |arXiv:math.AG/0207012.

[50] E. Witten, Nucl. Phys. B 403, 159 (1993) arXiv:hep-th/9301042.

[51] J. de Boer, K. Hori, H. Ooguri, Y. Oz and Z. Yin, Nucl. Phys. B 493, 148 (1997) arXiv:hep-th/9612131.

[52] J.P. Gauntlett, G.W. Gibbons, G. Papadopoulos, and P.K. Townsend, Nucl. Phys. B 500, 133 (1997) arXiv:hep-th/9702202;

[53] R. Portugues, JHEP 0403, 051 (2004) [arXive: hep-th/0311099].

[54] N. S. Manton, Nucl. Phys. B 150, 397 (1979).

[55] R. Portugues and P. K. Townsend, Phys. Lett. B 530, 227 (2002) arXiv:hep-th/0112077.

[56] M. F. Atiyah and N. J. Hitchin, Phys. Lett. A 107, 21 (1985); Phil. Trans. Roy. Soc. Lond. A 315, 459 (1985).

[57] I. Affleck, M. Dine and N. Seiberg, Nucl. Phys. B 241, 493 (1984). 